\title{
Fully resolved simulation of particle deposition and heat transfer in a differentially heated cavity.
}

\author{
S. Haeri, J. S. Shrimpton \\ Tizzard Building, Faculty of Engineering and the Environment, Highfield Campus, \\ University of Southampton, Southampton, S017 1BJ, UK
}

\begin{abstract}
In this paper a fictitious domain method is used to study the motion of particles in a differentially heated cavity. A collision strategy is implemented which is validated using the problem of two freely falling particles with natural convection taking place from the leading hot particle. The motion of the particles in a differentially heated cavity is considered where the vertical walls are subject to a temperature difference $\Delta T$ whereas horizontal walls are assumed to be adiabatic. Depending on the fluid Grashof number different flow regimes and two critical Grashof numbers are identified. Sustained motion of the suspended particles is also studied and different behaviour is observed compared to the limiting case of tracer particles where simulations are usually performed using one-way coupled point-particle assumptions. Finally the effects of the particles on the heat transfer from the hot wall are studied and it is found that addition of large particles can adversely influence the heat transfer rate. However, if hot particles are effectively removed from the wall, e.g by increasing the Grashof number, wall heat transfer properties
\end{abstract}

Email address: John.Shrimpton@soton.ac.uk (J. S. Shrimpton)

Preprint submitted to International Journal of Heat and Fluid Flow January 28, 2014 
can still be enhanced.

Keywords: Particulate flow, Fictitious Domain, GPU acceleration, Differentially heated cavity, Natural convection, Direct Numerical Simulation

\section{Introduction}

Understanding the fundamentals of particle motion in a cavity can benefit many environmental and industrial applications. The main concern in this paper is the motion induced by the natural convection due to a temperature gradient which is particularly important in the environmental systems, chemical and biochemical reactors. For example, in clean rooms, understanding the patterns of motion is crucial for controlling the concentration and effective removal of the environmental pollutants such as dust, aerosol particles and chemical vapours. Another important application of flow induced in enclosures due to an internal or external heat source, is the prediction of heat loss in solar collectors [3] and double-glazed windows [18]. In addition, assessing the risk and environmental impacts of severe accidents in chemical or nuclear reactors is only possible by understanding the deposition and removal mechanisms of micron-sized particles from a buoyancy induced flow in large enclosures $[17,22]$.

A fundamental study of any of the aforementioned applications naturally reduces to the particle motion is a differentially heated cavity. A differentially heated cavity (DHC) is defined here as an enclosure filled with a fluid with a temperature dependent density, where the vertical walls are kept at two different, fixed temperatures denoted by $T_{h}$ and $T_{c}$ and the horizontal 
walls are assumed to be adiabatic. Fluid motion in a DHC has been studied extensively and many benchmark solutions are available, e.g De Vahl Davis [4] studied the 2D cavity problem and provided benchmark solutions for the laminar region for Rayleigh numbers between $10^{3}$ to $10^{6}$. More recently accurate results were provided by Le Quere [20] using a pseudo-spectral method for Rayleigh numbers between $10^{6}$ and $10^{8}$ by solving the Navier-Stokes and energy equations written in primitive variables.

Puragliesi et al. [22] studied the particle transport in a buoyancy driven flow in a DHC. They studied the turbulent flow at two different Rayleigh numbers $\left(R a=10^{9}\right.$ and $\left.10^{10}\right)$ using a Boussinesq approximation to include the density variation by the temperature gradient. They included the drag, gravity, buoyancy, lift and thermophoretic forces on the particle to calculate its motion. An Eulerian-Lagrangian (EL) approach is used to simulate the particle motion with a one-way coupled point-particle assumption. They found out that particle deposition is mainly caused by gravitational settling and the deposition mainly takes place at the bottom wall for particles with diameters larger than $10 \mu m$.

Similar to Puragliesi et al. [22], Akbar et al. [1] used a one-way coupled point-particle assumption for the simulation of particle motion in a DHC. However they used lower Rayleigh numbers in the range of $10^{2}$ to $8 \times 10^{5}$, and also considered the effects of the Brownian motion. They suggested that for the Rayleigh numbers larger than $10^{4}$ only one large recirculation pattern is observed for the motion of the particles. However for smaller Ra most particles disperse toward the walls while some of them are trapped in a recirculation zone. They may however, leave the recirculation zone due to Brownian 
motion at a very low rate. They also confirmed that the thermophoretic and Brownian motion are only important for sub-micron particles.

Heat transfer augmentation in a DHC was investigated by Tiwari and Das [24] for nano-particles and they reported a non-linear increase in the average wall Nusselt number by increasing the volume fraction. Recently Kuerten et al. [19] reported that the average wall Nusselt number can increase for millimetre size particles similar to the nano-particles. They reported enhancements as large as 100\% for heavy inertial particles.

All the aforementioned studies are performed using a point-particle assumption with one- or two-way coupling strategies. This approach cannot be classified as a full direct numerical simulation (DNS) since in either case inter-phase momentum and thermal transfers are modelled. The effects of particles on the motion of fluid is assumed to be negligible in a one-way coupled simulation. In the case of a two-way coupled simulation, an undisturbed flow field is assumed to calculate the forces [11, 12] which is then fed back to the Navier-Stokes (NS) equations using an averaging process. Therefore these methods are DNS only in the sense of the fluid motion. In this paper a fictitious domain method (FDM) [15] is used for the simulation of the particulate phase where no additional assumption is required for the calculation of the particle motion.

We first discuss the mathematical formulation and the numerical method succinctly. Although the method is based on a FDM which has been extensively validated elsewhere [13-15], a collision strategy is implemented and validated in this paper using the problem of two particles settling in a cavity with an aspect ratio of larger than one. In addition the linear solver 
is accelerated on GPGPU (General-purpose graphics processing unit) using cuSPARSE and cuBLAS libraries and the speed-up results are presented. Particle transport in a buoyancy driven cavity is then examined using the FDM. However, it should be noted that all the simulations in this paper are restricted to 2D cavities. Particles are initially at rest on the bottom of the cavity and we have identified three different regimes depending on the Grashof number. In the sustained circulation regime, it is found that a strong circulation area forms near the hot wall due to the body forces generated from the particles falling away from the hot wall. In addition the gravitational force prevents the particles to migrate to the weaker circulation areas near the cold wall. It is tested to confirm that this motion is independent of the initial configuration of the particle by simulating a randomly injected initial configuration. Finally effects of particles on the heat transfer

properties of the hot wall is studied and it is found that sluggish motion of large particles due to buoyancy and inertial effects can have a negative influence on the local value of the wall Nusselt number. However it is found that effective circulation of the particles, e.g. by increasing the Grashof number, can still enhance the wall Nusselt number.

\section{The Numerical Method}

\subsection{Governing equations}

The numerical method has been explained and validated extensively elsewhere [13-15]. However since a collision strategy is implemented and validated in this paper, the numerical method is discussed succinctly here. If we let $\Omega_{p}$ and $\Omega_{f}$ be the immersed particle and the fluid domain respectively 
and $\Omega=\Omega_{p} \cup \Omega_{f}$ be the domain including both the fluid and the particle, it is possible to extend the governing equations on the fluid domain (which will be the Navier-Stokes equations) to the particle domain by constraining the motion inside the particle domain to a rigid motion [21], i.e. by enforcing

$$
\frac{1}{2}\left(\frac{\partial u_{i}}{\partial x_{j}}+\frac{\partial u_{j}}{\partial x_{i}}\right)=0 \quad \text { in } \quad \Omega_{p} .
$$

Then the flow on the whole computational domain $\Omega$, is governed by the following continuity, momentum and energy balance equations:

$$
\begin{gathered}
\frac{\partial \rho}{\partial t}+\frac{\partial \rho u_{j}}{\partial x_{j}}=0, \\
\frac{\partial \rho u_{i}}{\partial t}+\frac{\partial \rho u_{i} u_{j}}{\partial x_{j}}=-\frac{\partial \sigma_{i j}}{\partial x_{j}}+f_{B, i}+f_{F D, i} . \\
\frac{\partial \rho c_{p} T}{\partial t}+\frac{\partial \rho u_{j} c_{p} T}{\partial x_{j}}=\frac{\partial}{\partial x_{j}}\left(\kappa \frac{\partial T}{\partial x_{j}}\right),
\end{gathered}
$$

where

$$
\sigma_{i j}=-P \delta_{i j}+\tau_{i j}, \quad \tau_{i j}=\mu\left(\frac{\partial u_{i}}{\partial x_{j}}+\frac{\partial u_{j}}{\partial x_{i}}\right),
$$

and $f_{F D, i}$ is a body force to impose the rigid motion. The buoyancy effects are presented by $f_{B, i}$, the fluid pressure by $P$ and $u_{i}$ is the fluid velocity. The mixture density, heat capacity and thermal conductivity are represented by $\rho, c_{p}$ and $\kappa$ respectively. Mixture properties are defined by

$$
\varphi=\Theta_{p} \varphi_{p}+\left(1-\Theta_{p}\right) \varphi_{f}
$$

where $\varphi=\left\{\rho, c_{p}, \kappa\right\}$ and subscripts $p$ and $f$ are used to refer to the particle and fluid properties at some reference temperature respectively. Also note that the tensor notation is used in this paper; for example a variable with subscript $f, i$ is vector variable in fluid domain. and $\Theta_{p}$ is a step function that 
takes the value of one in $\Omega_{p}$ and zero otherwise. In the current method the particles are defined by an explicit stair-step grid (material grid hereafter) which freely moves on the background Eulerian grid where the solution is sought. Figure 1 shows the material grid points used to define the particle. Using this explicit grid, particle volume fractions on the background Eulerian grid can efficiently be calculated by using the discrete delta functions [15]:

$$
\Theta_{p}(\mathbf{x})=\sum_{m=1}^{N_{m}} \delta_{h}\left(\frac{\mathbf{x}-\mathbf{X}_{m}}{h}\right) v_{m} \quad \forall \mathbf{x} \in g_{h} .
$$

where $N_{m}, v_{m}$ and $g_{h}$ are the number of material control volumes, volume of the m-th material $\mathrm{CV}$ and the support of the discrete delta function respectively. Also note that $\mathbf{X}_{m}$ refers to the position of the $m$-th material grid point. The discrete delta function, $\delta_{h}$, is defined by

$$
\delta_{h}(\mathbf{r})=\frac{1}{h^{d}} \prod_{i=1}^{d} \phi\left(r_{i}\right)
$$

where $d$ is the dimensionality of the Eulerian grid, $h$ is the Eulerian grid spacing and $r_{i}=\left(x_{i}-X_{m, i}\right) / h$. There are several choices for the function $\phi(r)$ in Eq. (8), one such function with reasonable smoothing and computational efficiency [13] is the following function first suggested by Roma et al. [23]:

$$
\phi(r)= \begin{cases}\frac{1}{3}\left(1+\sqrt{1-3 r^{2}}\right), & |r| \leq 0.5 \\ \frac{1}{6}\left(5-3|r|-\sqrt{1-3(1-|r|)^{2}}\right), & 0.5<|r| \leq 1.5 \\ 0, & |r|>1.5\end{cases}
$$

The buoyancy force $f_{B, i}$ in Eq.(3) can be written by [15]:

$$
f_{B, i}=\left(\Theta_{p}\left(\rho_{p_{T}}-\rho_{f}\right)+\left(1-\Theta_{p}\right)\left(\rho_{f_{T}}-\rho_{f}\right)\right) g_{i}
$$


where subscript $T$ is used to refer to the current temperature. Using a Boussinesq approximation, Eq.(10) can be rearranged to get the following equation for the total body force

$$
f_{B, i}=-\left(\Theta_{p} \beta_{p} \rho_{p_{T}}+\left(1-\Theta_{p}\right) \beta_{f} \rho_{f_{T}}\right)\left(T-T_{r e f}\right) g_{i}+\left(\rho-\rho_{f}\right) g_{i} .
$$

In Eq.(11), $\beta$ is the coefficient of volumetric expansion which is a constant for each phase.

\subsubsection{Source correction}

The SIMPLE $[5,26]$ algorithm on collocated grid is used for the pressure velocity coupling which is an iterative process. A second order backward difference in conjunction to the implicit Euler method are used for the time integration of the temporal and spacial terms respectively [15]. Denoting the predicted value of the body force at the current iteration by $f_{F D, i}^{*}$ a correction force $f_{F D, i}^{\prime}$ can be defined such that $f_{F D, i}^{*}+f_{F D, i}^{\prime}$ is the required force to impose the rigidity constraint in the particle domain at the current time step. This correction force can be written by [15]:

$$
f_{F D, i}^{\prime}(\mathbf{x})=\sum_{m=1}^{N_{m}} F_{i}^{F D}\left(\mathbf{X}_{m}\right) \delta_{h}\left(\frac{\mathbf{x}-\mathbf{X}_{m}}{h}\right) v_{m} \quad \forall \mathbf{x} \in g_{h}
$$

The required body force on the material grid points $F_{m, i}^{F D} \equiv F_{i}^{F D}\left(\mathbf{X}_{m}\right)$, can be calculated by

$$
F_{m, i}^{F D}=\rho_{p} \frac{U_{m, i}^{R}-U_{m, i}^{*}}{\Delta t}, \quad 1 \leq m \leq N_{m}
$$

where

$$
U_{m, i}^{*}=\sum_{\mathbf{x} \in g_{h}} u_{i}^{*}(\mathbf{x}) \delta_{h}\left(\frac{\mathbf{x}-\mathbf{X}_{m}}{h}\right) h^{d}, \quad 1 \leq m \leq N_{m}
$$


and $u_{i}^{*}$ are the fluid velocities at the current iteration. The rigid velocity of the individual material points $U_{m, i}^{R}$ is given by [15]:

$$
U_{m, i}^{R}=U_{p, i}+\epsilon_{i j k} \omega_{p, j}\left(X_{m, k}-X_{p, k}\right), \quad 1 \leq m \leq N_{m}
$$

where linear $\left(U_{p, i}\right)$ and angular $\left(\omega_{p, i}\right)$ velocities of the particle are calculated from $U_{m, i}^{*}$ using the principles of conservation of angular and linear momen-

tum, see [15] for details. Also note that the a subscript 'p' $\left(1 \leq p \leq N_{p}\right)$ refers to the particle centres whereas a subscript 'm' $\left(1 \leq m \leq N_{m}\right)$ refers to the material points forming that particle. In Eq.(15), $\epsilon_{i j k}$ is the usual permutation symbol which is equal to 1 for even permutations of $i j k,-1$ for odd permutations and 0 otherwise. The freely varying particle temperature is obtained directly from the solution of Eq.(4) and no further treatment is required.

\subsection{Collision strategy}

When considering more than one particle or when a particle comes too close to a wall, a forcing term should be implemented to prevent particles from overlapping other particle domains or penetrating the domain walls. Simulating the particle collision in fully-resolved methods is the subject of significant research. A widely used method to consider particle-particle collision or particle-wall collision is to calculate the distance between each pair of particles after the particle positions are updated. Then a type of short range repulsive force is calculated for the $p$ th particle by

$$
F_{p, i}^{C}=\sum_{\substack{q=1 \\ q \neq p}}^{N_{p}} F_{q p, i}+\sum_{w=1}^{N_{w}} F_{w p, i}
$$


where $N_{w}$ is the number of domain walls and $N_{p}$ is the number of particles in the domain. Superscript $C$ is used to indicate the sum of all collision forces on particle $p$ and subscript $\ell p, i$ is used to indicated the collision force in direction $i$ exerted by object $\ell$ on particle $p$. Object $\ell$ can be another particle or a wall which are indicated by $q$ and $w$ respectively. The collision forces $F_{\ell p, i}$ on $p$-th particle can be modelled using a short range repulsive force which following Glowinski et al. $[6,7,8]$ can be defined by:

$$
F_{q p, i}=\xi_{q p}\left(X_{q, i}-X_{p, i}\right)\left(\max \left[0, \frac{D_{q}+D_{p}}{2}+\Delta-d_{q p}\right]\right)^{2},
$$

where $d_{q p}$ is the distance between particle pair $(q, p)$ and $\Delta$ is the range of activation of the force. Particle-wall collisions are determined by creating a mirror image $p^{\prime}$ of the particle $p$ on the other side of the wall, i.e $F_{w p, i}=F_{p^{\prime} p, i}$. Note that the adjustable parameter $x i_{p q}$ is dimensional and has a dimension of force per unit volume. In this study identical values are used for the dimensional adjustable parameters $\xi_{q p}$ for all the particle pairs. Despite the easy implementation of Eq.(17), there are generally a few issues associated with it. First issue is that the adjustable parameter is problem dependent and is not known a priori. However, a series of numerical experiments which will be detailed in Section 3.1, show that a value of $\xi_{q p}=2 \times 10^{6}$ can be used for the simulations in this paper for a particle density of $\rho_{p}=1.1$ and collision Stokes numbers of $S t \geq 10$, where no rebound occurs and all the particle kinetic energy dissipates in fluid $[2,10]$. The collision Stokes number $S t$ is defined by $[10]$

$$
S t=\frac{1}{9}\left(\frac{\rho_{p}}{\rho_{f}}\right) R e
$$


with $R e=\rho_{f}\left|U_{p}-U_{f}\right| D_{p} / \mu_{f}$. This value is in the range of values $\left[10^{5}, 10^{7}\right]$ used in other immersed boundary (IB) [25] and FD simulations $[7,8]$. It is worth mentioning again that the value of $\xi_{p q}$ is problem dependant and is adjusted for the current simulations. However, if for a particular problem one sets the value of adjustable parameter such that for $S t \approx 10$ no rebound occurs then that can be regarded as the correct physics of the problem. In addition, if collision is of the main concern then more accurate (and more expensive) models such as those suggested in [16] should be adopted.

Second issue is that the range of activation of the repulsive forces should be chosen carefully in the current FD method. The choice of $\Delta$ is restricted by the support of the delta function used in this study. In the current method both the rigidity constraint and the divergence free criteria are corrected and therefore both criteria are fulfilled to the required precision. Therefore a slight overlap of the domains is equivalent to requiring the fulfilment of two different constraints at the same grid point which causes the failure of the iterative process. Therefore a value of $\Delta=3 h$ is used in this study. Finally, this repulsive forcing function is continuous and its value grows as $d$ decreases, where $d$ is the separation distance defined in Eq.(17). Therefore using this equation, requires very small time steps such that the force increases continuously in a few steps. Required step sizes, are prohibitively small due to the iterative nature of the current implementation. Therefore the following integration technique is adopted for the current method.

In this approach first the time step is divided into a number of smaller sub-steps using $\Delta t_{k}=\Delta t / N_{k}$ where $N_{k}$ is the number of sub-steps. The collision force is then calculated by first calculating the movement of each 
particle using

$$
X_{p, i}^{k}=X_{p, i}^{k-1}+U_{p, i}^{k} \Delta t_{k}
$$

where the superscript $k=1$ corresponds to the values at the previous time step $(n-1)$ and $k=N_{k}$ corresponds to the updated values at the current time step $(n)$. Also note that $X_{p, i}$ refers to the centre of the individual particles which is different from $X_{m, i}$ used to define the centre of the material grid points that constitute each particle. In addition in this study we only consider circular particles and hence, only the particle centres are considered to identify the colliding pairs. Total collision force for each particle is then calculated using Eq.(16) and Eq.(17). The position of the particle is corrected using the calculated force by

$$
X_{p, i}^{k}=X_{p, i}^{k-1}+\frac{1}{4 M_{p}}\left(F_{p, i}^{C, k}+F_{p, i}^{C, k-1}\right) \Delta t_{k}^{2}
$$

and the velocities by

$$
U_{p, i}^{k}=U_{p, i}^{k-1}+\frac{1}{2 M_{p}}\left(F_{p, i}^{C, k}+F_{p, i}^{C, k-1}\right) \Delta t_{k}
$$

where $M_{p}$ is the total particle mass. At the end of this time integration, total movement of the particle is calculated by $\Delta X_{p, i}=X_{p, i}^{N_{k}}-X_{p, i}^{n-1}$ and the particle velocities are updated by

$$
U_{p, i}^{n}=\frac{\Delta X_{p, i}}{\Delta t}
$$

These velocities are then used to calculate $U_{m, i}^{R}$ as explained in Section 2.1.1. The rigid velocities $U_{m, i}^{R}$, are then used to provide the initial estimates for the body forces $f_{F D, i}^{*}$, at the current time step using Eq.(13) which is projected onto the Eulerian grid using Eq.(12), similar to the correction forces. Note 
also that $U_{p, i}^{n}$ is calculated only once at the beginning of each time step using this procedure, however, during the iterative procedure it is calculated using the conservation of linear momentum as explained in Section 2.1.1. In addition the current collision model only exerts normal forces in the direction of the line connecting the centres of particles, therefore it does not directly influence the angular velocity of the particles $\omega_{p, i}$. In addition, the position of the material grid points $\mathbf{X}_{m}$ are updated using the particle centre position and the rotation tensor [9] which are updated after each time step [15].

The collision strategy is activated during the particle position update of the FD algorithm [15] and can be summarized as follows:

- For each particle 'p' identify the colliding pairs (i.e. the set of particles ' $\mathrm{q}$ ' for which $\left.\left(D_{q}+D_{p}\right) / 2+\Delta-d_{q, p}>0\right)$.

- Update the positions for sub time step $\Delta t_{k}$ using Eq. (19).

- Calculate the collision force between the colliding pair 'qp' using Eq. (16).

- Using the calculated collision force recalculate the centre position using Eq. (20) and velocities by Eq. (21).

- For the numerical consistency at the end of the final sub time step re-estimate the particle velocities using Eq. (22).

\section{Results and discussions}

The main simulations in this paper are time consuming and a parallelization strategy is required for the simulations to be feasible. In this paper 
GPGPU acceleration is considered for the parallelization of the code and the results are presented in Appendix A. Also note that, a CGS system of units is assumed in this paper except otherwise stated. In this section first the collision strategy explained in Section 2.2 is validated using the problem of two settling particles. The benchmark is also extended to consider the case where natural convection takes place from the leading particle. Then the motion of particles in a cavity is considered where critical Grashof numbers are identified and the effects of particle motion on the wall heat transfer properties are discussed.

\subsection{Particle-particle collision}

In this section sedimentation of two particles at a low Stokes number in a 2D channel filled with a Newtonian fluid is studied to validate the current particle collision strategy. A cavity with height 6 and width 2 is considered and the particle diameters are set to $D=0.2$ which are released at $y=0.5$ and $y=5.3$. Fluid density, density ratio, gravitational acceleration and fluid viscosity are set to $\rho_{f}=1, \rho_{p} / \rho_{f}=1.1, g=-981$ and $\mu_{f}=0.08$ respectively. These properties are commonly used for this problem and are adopted here merely to compare the results to other benchmark solutions $[2,25]$. The Reynolds number based on the fluid properties and maximum velocity of the trailing particle is 9.68 and the corresponding Stokes number is $S t=1.18$. Particles are initially displaced by $0.004 D$ as suggested in $[2,25]$ to let the instabilities to grow during the short collision time. Figure 3 summarizes the motion of the two particles during the course of collision. Results of the $U_{p}$ and $V_{p}$ are compared to the previous study [2] and very good agreement is observed before the collision. However, after the collision the results can 
only be compare qualitatively. It is discussed in [25] that the motion of the particle after the collision is a manifestation of the strong instabilities in the motion of particles and hence given different collision strategies and different numerical methods significant differences in the particle motion after the collision are expected, which is also reported in [2]. However, given that a different collision strategy is used in this study, the general behaviour of the curves after the collision are similar to those reported in [2] with only a time lag of about $4.91 \tau_{p}$ where $\tau_{p}=\rho_{p} D^{2} /\left(18 \mu_{f}\right)$.

Overtaking the trailing particle happens at $t=0.81$ and can be identified from the intersection of the two graphs in Figure 3(d). The base simulation is performed on a grid with uniform spacing, $h=1 / 256$, and a time step size of $\Delta t=0.002$. To examine the time and grid independence of the collision strategy two other grid resolutions with $h=1 / 192$ and $h=1 / 320$ and one smaller time step of 0.001 are used. In Figure 3 results of all these simulations are also presented. This shows that particle motion even after the collision is indeed, time and grid independent and only depends on the order of the accuracy of the method used. The number of sub time steps is set to $N_{k}=5$ in this study and the independence of the results from this choice is demonstrated by solving the same problem for the finest grid $(h=1 / 320)$ and $N_{k}=10$ and the results of this test are presented in Figure 4.

Similar problem is further considered by initializing the bottom particle to a hotter temperature, $T_{H}$, and allowing for natural convection from the particles. A Grashof number based on the particle diameter is defined by

$$
G r_{D}=\frac{\rho^{2}\left(T_{H}-T_{\infty}\right) g \beta_{f} D^{3}}{\mu^{2}} .
$$

A fine grid with $h=1 / 256$ and $\Delta t=0.002$ are used for this study. Four 
different Grashof numbers $G r_{D} \in\{100,500,1000,2000\}$ are considered by changing the $\beta_{f}$ and no thermal expansion is considered in the particle domain, i.e. $\beta_{p}=0$. Particles are initially displaced similar to the case of pure sedimentation discussed earlier in this section. All the other parameters remain the same and particle temperature varies freely in time. Figure 5 shows the variation of the mean temperature $\bar{T}$ defined by

$$
\bar{T}=\sum_{m=1}^{N_{m}} T_{m} v_{m} / \sum_{m=1}^{N_{m}} v_{m}
$$

for the leading and trailing particles in time. At lower Grashof numbers the trailing particle follows the leading particle for a longer time and hence the the temperature of the trailing particle exceeds that of the leading particle at $t=0.45$ for $G r_{D}=100$ and $t=0.53$ for $G r_{D}=500$. However for the larger Grashof numbers $G r_{D}=1000$ and $G r_{D}=2000$ the mean temperature of the trailing particle never exceeds that of the leading particle due to a fast upward motion induced by the natural convection from the bottom particle.

Figure 6 shows the time history of the position of the particles at the four different Grashof numbers. At the lowest Grashof number $G r_{D}=100$, the time history of the position of the particle does not significantly differ from the pure settling case presented in Figure 3. However at $G r_{D}=500$ no tumbling is observed. Figure 7 shows the z-vorticity contours superimposed on the temperature contours for the four different cases just before the first collision. At $G r_{D}=100$ only a single dominant vortex is observed which is formed due to the downward motion of the particles. In this case vortices do form due to the upward flow induced by natural convection however they form on top of the channel and have little impact on the motion of the particles. 
Figure 6(b) shows the time history of the particle positions at $G r_{D}=500$ where no tumbling is observed. This seems to be due to the formation of a pair of counter rotating vortices around the particles, see Figure 7(b), which are not strong enough to lift the leading particle and only have a stabilizing effect on the motion of the trailing particle. Therefore although collisions happen during the course of motion, these secondary vortices prevent the instabilities to grow. At $G r_{D}=1000$ and $G r_{D}=2000$, the secondary vortices are strong enough to lift the leading particle. Contrary to the case of $G r_{D}=$ 500 where both particles were actually settling in this case the leading particle is moving upward and is pushing the trailing particle up which is by nature unstable, see Figures 7(c) and 7(d). Therefore instabilities do grow in these two cases which results in tumbling of the particles and eventually the two particles separate, see Figures 6(c) and 6(d).

\subsection{Motion of particles in a cavity}

In this section, particle motion in a $2 \mathrm{D}$ DHC is studied in detail. A cavity with sides $L=1$ is considered and is discretized with $N=1000$ nodes in each coordinate directions. For the first test case three rows of identical particles are uniformly laid on the bottom wall giving a total of $N=129$ particles. Following physical properties (CGS system) are used for the test cases

- $\mu_{f}=0.01, \rho_{f}=1, \frac{\rho_{p}}{\rho_{f}}=1.1$,

- $\frac{\kappa_{f}}{\kappa_{p}}=\frac{1}{5}, c_{p f}=10, \frac{c_{p f}}{c_{p p}}=5$,

- $D_{p}=0.02, g=-981$.

A Grashof number $G r_{L}$, based on the cavity side length $L$ is defined similar to Eq.(23) with $D$ replaced by $L$ which determines the strength of the large scale 
flow induced by the natural convection. Also note that $T_{H}$ is the temperature of the hot wall in this case. The range of Grashof numbers considered here is comparable to the values used in [1] and [22] with air as the operating fluid. In addition, the gravitational effects are determined by the Archimedes number $A r=g D^{3} \rho_{f}\left(\rho_{p}-\rho_{f}\right) / \mu^{2}$, which specifies the fluidization and sedimentation behaviour of the particles, that are the main concerns of this paper. The Archimedes number for the current system is $A r=7.85$ which is comparable to $A r=3.27$ for $36 \mu m$ spherical particles used in [22]. It should, however, be noted that a full examination of all these parameters is outside the scope of the current paper and only the effects of the Grashof number is considered. The Prandtl number based on fluid properties $\operatorname{Pr}=c_{p f} \mu_{f} / \kappa_{f}$, is set to $\operatorname{Pr}=0.7$ in this study and is kept constant for all simulations. Rayleigh number $R a_{L}=\operatorname{Pr} \cdot G r_{L}$ can be used to summarize all the variable that affect the large scale motions, however since $\operatorname{Pr}$ is a constant in this study all the results are reported based on the $G r_{L}$.

We first investigate the motion of the particles at different $G r_{L}$ numbers. By performing several numerical experiments we have identified critical $G r_{L}$ numbers that significantly effect the patterns of particle motion. Figure 8(a) shows the bed of particles at the bottom of the cavity at $t=5$ after the start of the calculation with $G r_{L}=5 \times 10^{5}$. At this Grashof number the strength of the large scale eddies generated by natural convection is not enough to lift the particles. Although some particles still manage to escape the bed and move toward the hot wall, they will not move to the circulating flow. Figure $8(\mathrm{c})$ shows the temperature contours of the DHC at $t=5$. Particles in the region specified by a box on Figure 8(c) are enlarged in Figure 8(d). 
This figure clearly shows that at the bottom, due to the larger conductivity, $\kappa_{p}$, of the particles and smaller specific heat $c_{p p}$, a significant amount of heat diffuses into the bed via the near wall particles which significantly changes the local heat transfer behaviour. The particle effects on the heat transfer properties are further discussed in Section 3.3. Figure 8(b) shows the escape mechanism of the particles. It seems that one of the escape mechanisms is due to the net effects of two flow generated torques acting on the particle, one generated from the large scale eddies and one from the upward flow generated due to natural convection from the lower layers of hot particles. These two forces generate a rotation with an off-centre axis of rotation which can roll the particles up its neighbours.

The behaviour of the system remains the same up to $G r_{L}=10^{6}$ where lift forces induced by the large scale motions can lift the particles near the hot wall. To better present the behaviour of the system, the following averaging is performed on the position of the particles. The domain is divided into equal number of bins in $\mathrm{x}$ - and $\mathrm{y}$-directions and the number of particles in each bin is calculated at each time step. Then the number of particles in each bin is averaged during the sampling period $\left(t_{s}-t_{0}\right)$ to yield an average particle per cell defined by

$$
\bar{N}_{\text {bin }}=\frac{\sum_{t=t_{0}}^{t_{s}} N_{\text {bin }} \Delta t}{t_{s}-t_{0}},
$$

where $t_{0}, \Delta t$ and $N_{\text {bin }}$ are the time at the start of the sampling, time step size and the number of particles per bin at a specific time, respectively. Also note that 25 bins are initially allocated and the bins are located on the cell centres between $0.02 \cdots 0.98$. 
Figures 9(a) and 9(b) show the particle position and z-vorticity contours for $G r_{L}=10^{6}$ and $G r_{L}=2 \times 10^{6}$ at $t=20$ respectively. The first critical Grashof number is at $G r_{L}=10^{6}$ where a few particles escape the bed and move up the hot wall. However the large scale eddies are still not strong enough to sustain the particles in suspension and the particle start to fall on average at a distance of $L_{f} \approx 16 D_{p}$, see also Figure 9(c). In addition, note that the particles almost exactly follow the path of the leading particles due to a mechanism explained in Section 3.1. However examination of the local vorticity contours shows that no significant flow forms near the particles due to the natural convection. Figure 10 shows the temperature contours in a box specified in Figure 9(b). Particle specific heat is smaller than the fluid's in this study with a larger thermal conductivity $\left(\kappa_{p} / \kappa_{f}=5\right)$ which means that the particle quickly attains thermal equilibrium with the surrounding fluid and effectively push the temperature contours away from each other. Due to this local thermal equilibrium with the surrounding fluid, no significant local convection is observed. Although thermal properties of the particles may affect this behaviour no parametric study is performed in this paper. At larger $G r_{L}=2 \times 10^{6}$ similar behaviour is observed except that the falling distance of the particle can be as large as $L_{f} \approx 25 D_{p}$, see Figure $9(\mathrm{~d})$.

Streamlines in Figures 9(a) and 9(b) show that a local eddy can form due to the upward motion of the particles near the hot wall and the free fall of particles at the falling distance $L_{f}$. This local eddy gets stronger at $G r_{L}=2 \times 10^{6}$ and traps some of the particles. Therefore the expected number of particles is non-zero between the hot wall and the falling distance $L_{f}$ compared to the lower Grashof number case, see Figures 9(c) and 9(d). 
Note that in this case, $\bar{N}_{\text {bin }}$, is calculated on 21 initially allocated bins from $y=0.18$ in the $\mathrm{y}$-direction, which is the height of the settled particles. In addition, $\bar{N}_{\text {bin }}$ is further interpolated on a 4 times finer grid which is merely done for a better presentation.

Figure 11 shows four different stages of particle-particle collision for $G r_{L}=$ $2 \times 10^{6}$. Particles colliding on top of a row of particles (Particle labelled $B$ in Figure 11) exerts a normal force with no tendency to rotate particle $C$. However these settling particle have significant linear momentum which is transferred to the colliding particle (particle $C$ ) through a thin layer of fluid between the two particles which exerts a torque on particle $C$ and rotates this particle. It should be noted that only a normal force is modelled in this study and tangential forces are neglected. Therefore, in real physical system friction between neighbouring particles in the row, may quickly deteriorate or even prevent the rotation of particle $C$. In any case this type of collision does not seem to excite the particle to escape from the bed. However the particle settling at a lower layers (particle $A$ ) slide on these layers and collide with the row of particles just in front of them. This collision transfers linear momentum to the row which can excite new particle to escape the bed by generating an upward flow between the particle gaps. Note that the settling particles have both angular and linear momentums (both roll and slide), whereas the particles already on a row, mainly gain angular momentum due to the flow induced torques (only roll), see Figure 8(b).

The Grashof number is further increased to $G r_{L}=5 \times 10^{6}$ which is the next critical value for the Grashof number. Figure 12 shows the vorticity contours and average number of particles per cell for $G r_{L}=5 \times 10^{6}$ and 
$G r_{L}=10^{7}$. Note that in this figure the whole domain is represented since all particles are fluidized because of the strong large scale eddies. At $G r_{L}=$ $5 \times 10^{6}$ two distinct regions start to develop. A circulation region near the hot wall forms similar to the mechanism described for the lower Grashof number cases. Additionally a high concentration region is formed at the top left of the circulation region where some particles get trapped between a large negative vortex and the wall, See Figures 12(a) and 12(b). In addition, increasing the Grashof number seems to push the particles more toward the hot wall and more effectively circulates the particle specially on top left corner where particle motion becomes sluggish. This clustering of particles is significantly different from the results presented in earlier studies [1] where one-way coupled tracer particles are used. In the limiting case of tracer particle, they sample a larger segment of the cavity comparable to the size of the large scale eddies whereas here, particles accumulate near the hot wall due to the particle falling and drafting effects which can produce strong local eddies.

To further test if this clustering behaviour depends on the initial configuration of the bed we perform another test at $G r_{L}=5 \times 10^{5}$ with an initial population of randomly distributed particles (both in $\mathrm{x}$ - and $\mathrm{y}$-directions) in the domain. Figure 13 shows the particle distribution at three different times for this case. Evidently the initial configuration of the particles has no effect on the clustering behaviour and they still tend to cluster near the hot wall.

\subsection{Particle effects on the wall heat transfer properties}

In this section the effects of the particle motion on the heat transfer properties of the wall are studied. To assess the particle effects a wall Nusselt 
number is defined by

$$
N u_{w}=\left.\frac{L}{T_{h}-T_{c}} \frac{d T}{d n}\right|_{w},
$$

which is averaged for $t_{s}-t_{0}=1$, to yield an average wall Nusselt number

$$
\overline{N u}_{w}=\frac{\sum_{t=t_{0}}^{t_{s}} N u_{w} \Delta t}{t_{s}-t_{0}} .
$$

Note that in Eq.(26), $n$ is the wall normal direction and the averaging time is chosen such that the particle circulate once around the local eddy. Figure 14 shows the local value of the Nusselt number $\overline{N u}_{w}$, along the vertical hot wall of the cavity. At $G r_{L}=5 \times 10^{5}$ a significant increase in the value of the local Nusselt number is observed where the particles reside. This is due to the fact that the local isotherms are pushed toward the hot wall which causes a large local temperature gradient, see Figure 8(d). However in the gaps between the particles the value of the local Nusselt number is significantly reduced to values even below that of an empty cavity. This phenomena is more pronounced in Figure 14(b) where a significant reduction in the heat transfer performance at $y<0.2$ is obvious. This due to the fact that the heat transfer mechanism is solely conduction dominated in this region whereas large scale eddies could remove a significant amount of heat from the wall if a heap of particles had not been accumulated on the bottom left corner. The local Nusselt number is further averaged along the hot wall to yield

$$
\overline{\overline{N u}}_{w}=\frac{\sum_{y=0}^{L} \overline{N u}_{w} \Delta y}{L} .
$$

Figure 15 shows the average Nusselt number defined by Eq.(28) for four different Grashof numbers. At $G r_{L}=5 \times 10^{5}$ although the heat transfer in the particle heap is diffusion dominated, strong diffusion from the particles is 
more significant than the reduction in convective heat transfer. Additionally particles act as additional sources and the net effect is an increase in the total wall Nusselt number, see Figure 15. At $G r_{L}=2 \times 10^{6}$ this reduction in strong convective heat transfer is more important and hence the overall heat transfer rate is significantly reduced.

At two higher Grashof numbers this heap effects are limited to $y<0.05$ and hence do not seem to significantly affect the total Nusselt number. However as discussed in the previous section a high particle concentration region is formed on the top left corner of the cavity where particle become sluggish and similar to the heap effects reduce the eddy strength on that region. This adversely affects the Nusselt number in this region which can be seen in Figures $14(\mathrm{c})$ and $14(\mathrm{~d})$ at around $y=0.58$ and 0.64 respectively. Obviously this region is smaller and particle buoyancy effects are less significant for $G r_{L}=10^{7}$ and hence the total Nusselt number eventually increases, see Figure 15.

Heat transfer enhancement by adding nano-particles is a well known phenomena but recently it has also been reported for millilitre size particles [19]. This current results show that a significant flux of cold particles should be provided near the wall by the large scale flow and the particles should be effectively removed by the flow for a notable heat transfer augmentation to occur. In addition, for large size particles, particle buoyancy effects which causes accumulation in the corners, can adversely influence the heat transfer rate to an extent of actually decreasing the total Nusselt number. 


\section{Concluding remarks}

In this paper a novel fictitious domain method is used to study the fundamental mechanisms of the particle motion in a differentially heated cavity. A collision strategy is implemented and tested by considering the settling of two particles in a quiescent flow. This benchmark problem is extended by considering the natural convection from the hot leading particle and critical Grashof number, based on the particle diameter, are identified for this motion.

Motion of the particles in a cavity is then studied by considering three rows of particle initially laying on the bottom of the cavity. Three different flow regimes are identified depending on the Grashof number which is defined based on the domain length scale. Particle fluidization mechanism is studied and the roll of particle collision in exciting new particles to scape the bed is discussed in detail by considering different types of particle-particle collisions. Additionally it is found that the body forces induced by particles settling in large scale eddies can generate smaller local eddies which in turn cause the particles to accumulate near the hot wall and almost certainly will always remain in one half of the cavity. Finally the augmentation mechanism is explained in detail and it is shown that for large particles adding particle may adversely affect the heat transfer properties of the wall.

It should be noted that fully resolved simulations, despite using a small number of particles and utilizing new technologies, is still expensive for industrial scale simulations. The GPGPU parallelization used in this study provides a speed-up of 3-6 on different systems. However the speed-up presented in this paper suggests that with this new technology, fully resolved 
methods can be used for laboratory scale simulation if combined with conventional domain decomposition techniques. These techniques combined by a hierarchical modelling strategy seems to be the best approach for large scale simulations in the foreseeable future, c.f. Haeri and Shrimpton [11] for a suggestion on such hierarchies.

\section{Acknowledgement}

The authors acknowledge the use of the IRIDIS-3 High Performance Computing Facility, and associated support services at the University of Southampton, in the completion of this work.

\section{References}

[1] Akbar, M.K., Rahman, M., Ghiaasiaan, S.M., 2009. Particle transport in a small square enclosure in laminar natural convection. Aerosol Science 40, 747-61.

[2] Ardekani, A.M., Dabiri, S., Rangel, R.H., 2008. Collision of multiparticle and general shape objects in a viscous fluid. Journal of Computational Physics 227, 10094-107.

[3] Buchberg, H., Catton, I., Edwards, D., 1976. Natural convection in enclosed spaces - a review of application to solar energy collection. ASME Journal of Heat Transfer 98, 182-8.

[4] De Vahl Davis, G., 1983. Natural convection of air in a square cavity: a benchmark numerical solution. Int. J. Numer. Methods Fluids 3, 249-64. 
[5] Ferziger, J.H., Peric, M., 2002. Computational Methods for Fluid Dynamics. Springer.

[6] Glowinski, R., Pan, T., Hesla, T., Joseph, D., Priaux, J., 2001. A fictitious domain approach to the direct numerical simulation of incompressible viscous flow past moving rigid bodies: Application to particulate flow. Journal of Computational Physics 169, 363-426.

[7] Glowinski, R., Pan, T.W., Hesla, T., Joseph, D., Periaux, J., 2000. A distributed lagrange multiplier fictitious domain method for the simulation of flow around moving rigid bodies: application to particulate flow. Comput. Methods Appl. Mech. Engrg 184, 241-67.

[8] Glowinski, R., Pana, T.W., Hesla, T., Joseph, D., 1999. A distributed lagrange multiplier/fictitious domain method for particulate flows. International Journal of Multiphase Flow 25, 755-94.

[9] Goldstein, H., Poole, C., Safko, J., 2000. Classical Mechanics. Addison Wesley.

[10] Gondret, P., Lance, M., Petit, L., 2002. Bouncing motion of spherical particles in fluids. Physics of fluids 14, 643-52.

[11] Haeri, S., Shrimpton, J., 2011. A mesoscopic description of polydispersed particle laden turbulent flows. Progress in Energy and Combustion Science 37, 716-40.

[12] Haeri, S., Shrimpton, J., 2012a. Closure of non-integer moments arising in multiphase flow phenomena. Chemical Engineering Science 75, 42434. 
[13] Haeri, S., Shrimpton, J., 2012b. On the application of immersed boundary, fictitious domain and body-conformal mesh methods to many particle multiphase flows. International Journal of Multiphase Flow 40, $38-55$.

[14] Haeri, S., Shrimpton, J.S., 2013a. A correlation for the calculation of the local nusselt number around circular cylinders in the range $10 \leq$ $R e \leq 250$ and $0.1 \leq \operatorname{Pr} \leq 40$. International Journal of Heat and Mass Transfer 59, 219-29.

[15] Haeri, S., Shrimpton, J.S., 2013b. A new implicit fictitious domain method for the simulation of flow in complex geometries with heat transfer. Journal of Computational Physics 237, 21-45.

[16] Kempe, T., Frohlich, J., 2012. Collision modelling for the interfaceresolved simulation of spherical particles in viscous fluids. J. Fluid Mech , 445-89.

[17] Kissane, M.P., 2008. On the nature of aerosols produced during a severe accident of a water-cooled nuclear reactor. Nucl Eng Des 238, 2792-800.

[18] Korpela, S.A., Lee, Y., Drummond, J.E., 1982. Heat transfer through a double pane window. Journal of Heat Transfer 104, 539-44.

[19] Kuerten, J.G.M., van der Geld, C.W.M., Geurts, B.J., 2011. Turbulence modification and heat transfer enhancement by inertial particles in turbulent channel flow. Phys. Fluids 23, 123301-8.

[20] Le Quere, P., 1991. Accurate solutions to the square thermally driven cavity at high rayleigh number. Comput. Fluids 20, 29-41. 
[21] Patankar, N., Singh, P., Joseph, D., Glowinski, R., Pan, T.W., 2000. A new formulation of the distributed lagrange multiplier/ fictitious domain method for particulate flows. International Journal of Multiphase Flow $26,1509-24$.

[22] Puragliesi, R., Dehbi, A., Leriche, E., Soldati, A., Deville, M., 2011. Dns of buoyancy-driven flows and lagrangian particle tracking in a square cavity at high rayleigh numbers. International Journal of Heat and Fluid Flow 32, 915-31.

[23] Roma, A., Peskin, C., Berger, M., 1999. An adaptive version of the immersed boundary method. J. Comput. Phys 153, 509-34.

[24] Tiwari, R.J., Das, M.K., 2007. Heat transfer augmentation in a twosided lid-driven differentially heated square cavity utilizing nanofluids. International Journal of Heat and Mass Transfer 50, 2002-18.

[25] Uhlmann, M., 2005. An immersed boundary method with direct forcing for the simulation of particulate flows. Journal of Computational Physics 209, 448-76.

[26] Versteeg, H., Malalasekra, W., 2007. An Introduction to Computational Fluid Dynamics: The Finite Volume Method. Prentice Hall.

[27] van der Vorst, H.A., 1992. Bi-cgstab: A fast and smoothly converging variant of bi-cg for the solution of nonsymmetric linear systems. SIAM J. Sci. and Stat. Comput. 13, 631-44. 


\section{Appendix A. Code acceleration on GPU}

The first step in a GPGPU parallelization strategy is to time the code and identify the possible candidates for the GPU acceleration. Timing is performed using the code profiling tools provided by the Intel Parallel Studio and the DHC problem, which is discussed in detail in Section 3.2. Figure A.1 shows the results of the code timing. Clearly the single function that can benefit the most from the GPU acceleration is the linear solver which approximately consumes $90 \%$ of the total CPU time.

In this study a Krylov sub-space type iterative solver namely BiCGSTAB [27], is used to solve the linear equations resulting from the discretization of the momentum, pressure and temperature equations. The main algorithm is implemented using the $\mathrm{C}$ language and the cuSPARSE and cuBLAS libraries. Fortran 2003 iso_c_binding feature is used to interface the C function and to call the solver from the main Fortran code. The first test is performed on the Iridis- $3^{1}$ compute nodes which are equipped with $2.4 \mathrm{GHz}$ Intel Xeon E5645 processors and NVIDIA Tesla 20-series, M2050, GPGPU co-processors and approximately $22 \mathrm{~GB}$ memory. The second test is performed on the Emerald GPU cluster ${ }^{2}$. The Emerald comprises 60 HP SL390 compute nodes with two 6-core X5650 Intel Xeons, three 512-core M2090 NVIDIA GPUs and 48GB of memory, in addition 24 high memory nodes with 96GB memory and eight M2090 NVIDIA GPUs are available. The last test is performed on an Intel

\footnotetext{
${ }^{1}$ University of Southampton supercomputer facility ranked 331 on Top500 list on November 2012.

${ }^{2}$ Emerald is a large HP GPU system utilising 372 NVIDIA Tesla processors hosted at the University of Oxford.
} 
Core i7-2600 workstation with a midrange GeForce GTX $550 \mathrm{Ti}$ and 16Gb of memory. The Cuda SDK 4.2.9 and the Intel Fortran Compiler are used for compiling and linking the code on both systems. All the test on each system are performed with exactly the same set of compiler options, namely -03, -xHost, -no-prec-div and -ip. The results of this test is summarized in Table A.1. Using this parallelization strategy speed-ups of 6 and 5.2 are observed on dedicated workstation class GPGPUs M2090 and M2050 respectively. In addition a speed-up of 3.2 is observed with a midrange graphic card for this specific problem. Figure A.2 shows the profiling results after applying the GPU acceleration on the Core i7 workstation where the time for the linear solver is reduced to $70 \%$ of the total computation time. In addition, only $1 \%$ of the running time is spent in transferring data between main memory and the GPU memory which is achieved by retaining the frequently used data on the GPU memory. In the accelerated code $10 \%$ of the time is spent in transfers of variables between the Lagrangian and the Eulerian grids (Eq.(14) and Eq.(12)) which obviously increases by increasing the number of particles. This operation is relatively simple and is a potential candidate for further GPU acceleration. 


\begin{tabular}{|cccc|}
\hline Test & CPU Time (Sec) & GPU/CPU Time (Sec) & Speed-Up \\
\hline X5650/Tesla M2090 & 5657.02 & 941.04 & 6.0 \\
E5645/Tesla M2050 & 6835.04 & 1319.14 & 5.2 \\
Core-i7/GF 550 Ti & 6693.79 & 2086.52 & 3.2 \\
\hline
\end{tabular}

Table A.1: Comparison of the running times and speed-ups for the accelerated code. 


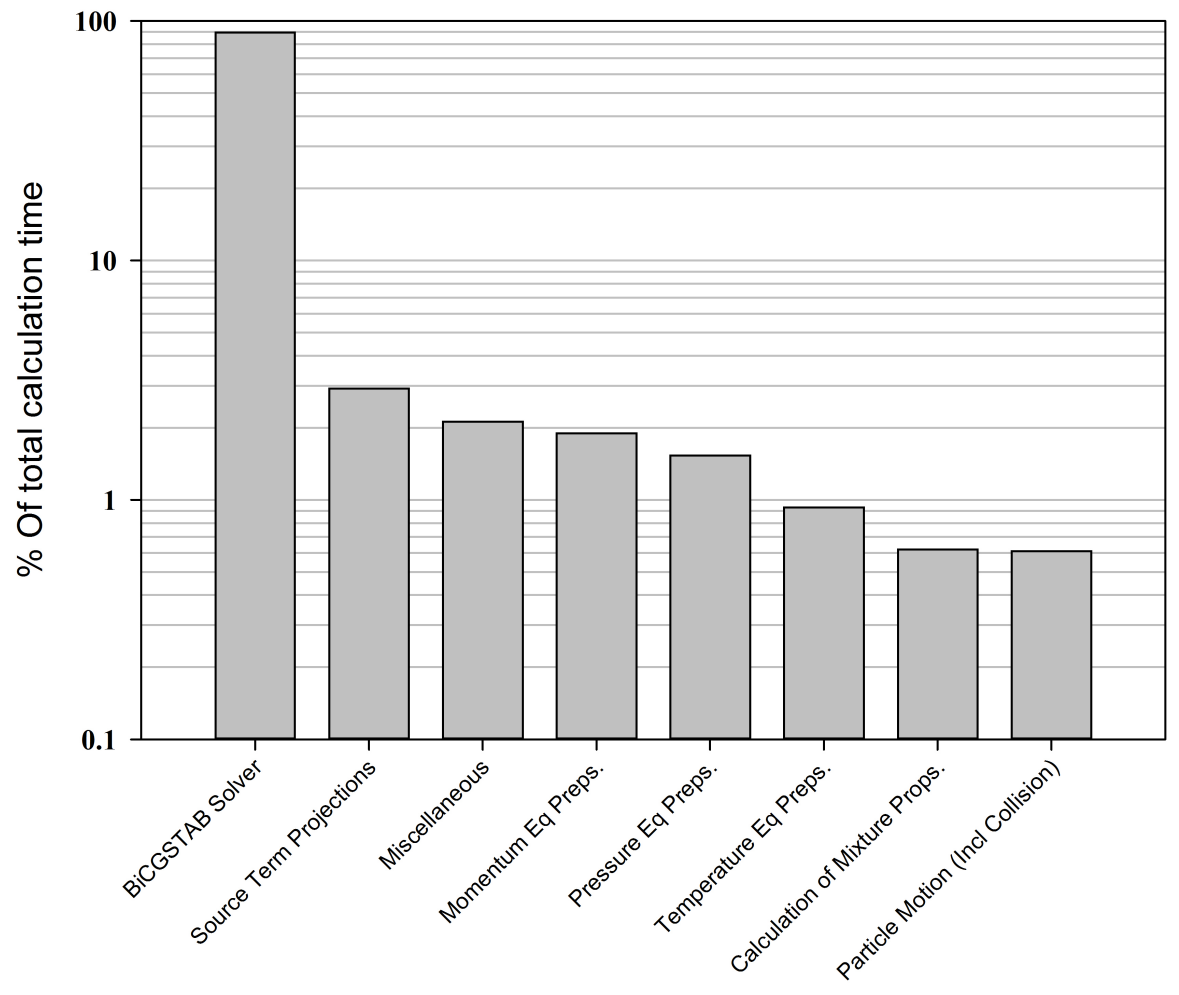

Fig. A.1: Time spent in different functions during the course of a sample run. $90 \%$ of the calculation time is spent in the iterative solution of the linear system using the BiCGSTAB solver. 


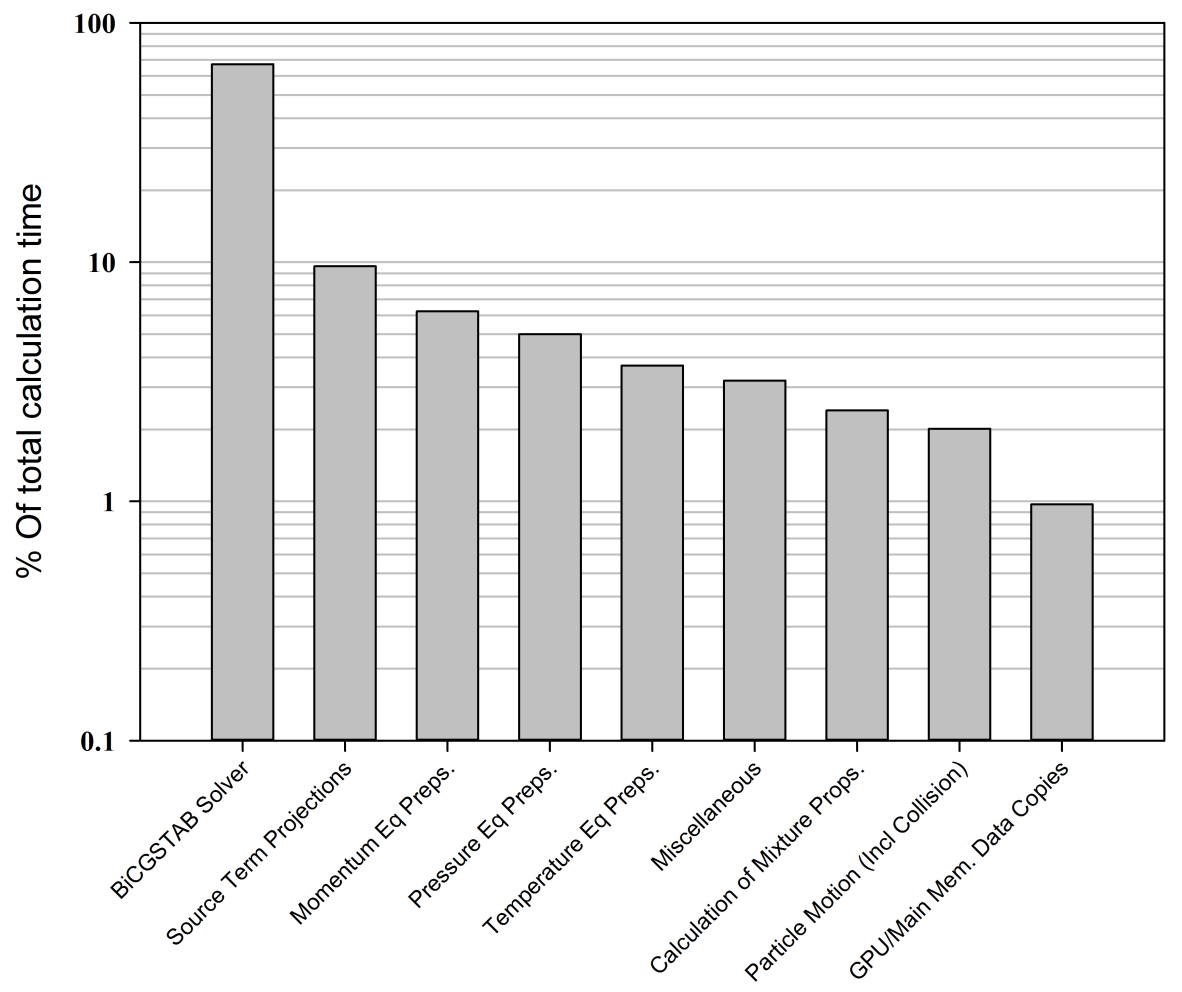

Fig. A.2: Time spent in different functions during the course of a sample run after GPU acceleration. 


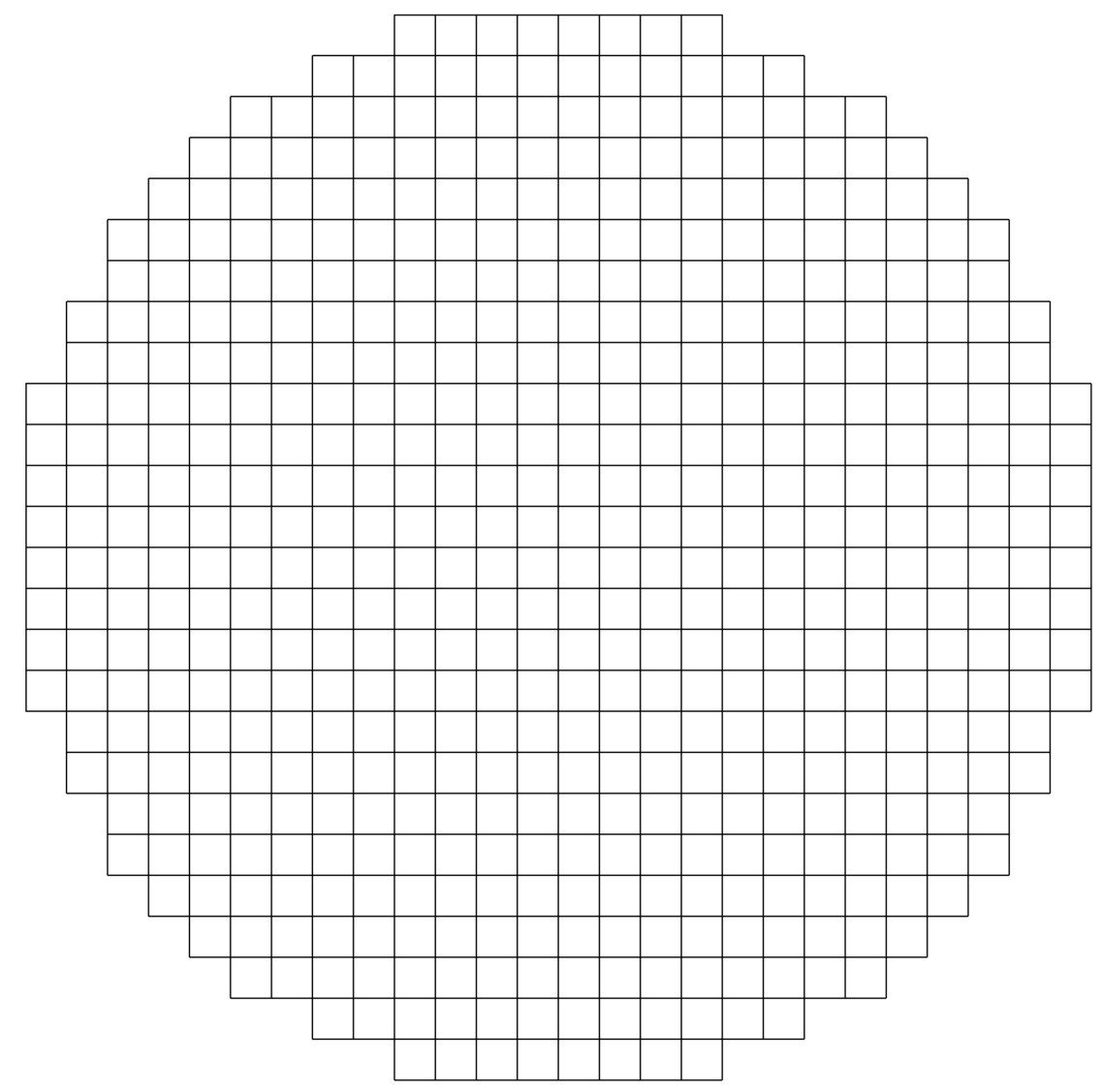

Fig. 1: Stair-step grid used for the discretisation of the particle. The depicted grid is much coarser than the actual grids used in the simulations. 


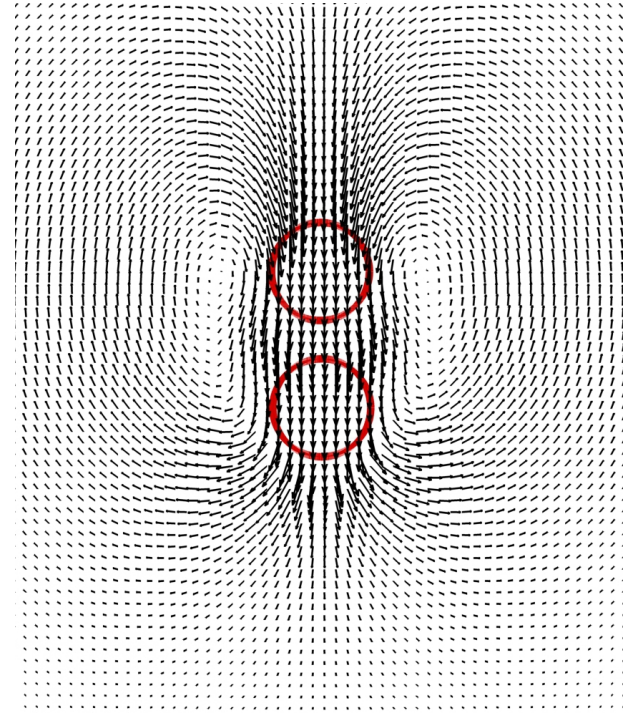

(a) $t=0.2$

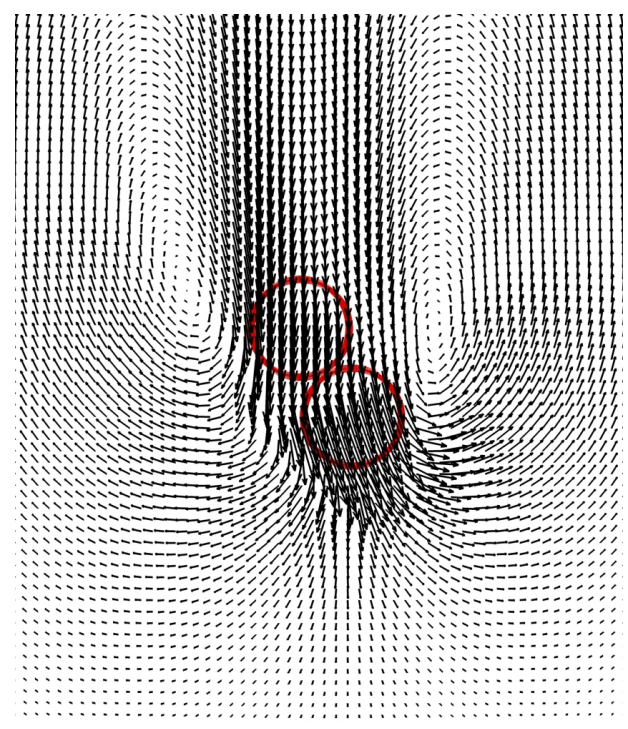

(c) $t=0.65$

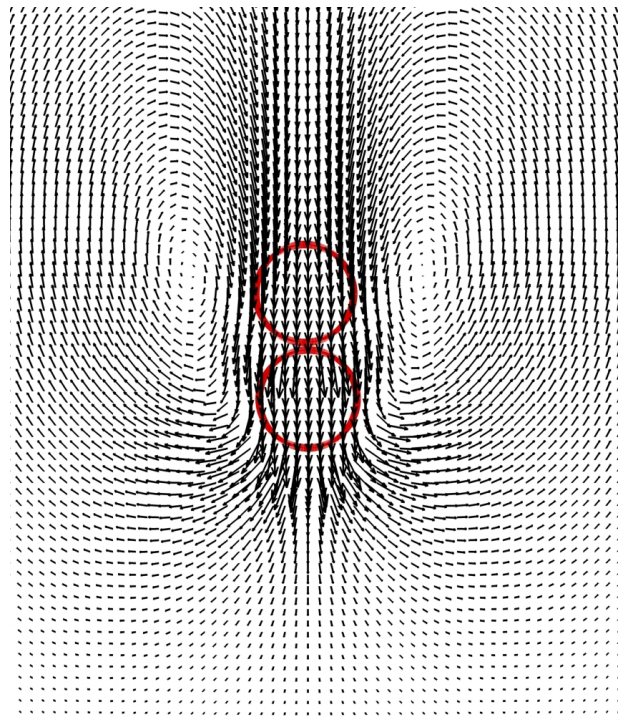

(b) $t=0.35$

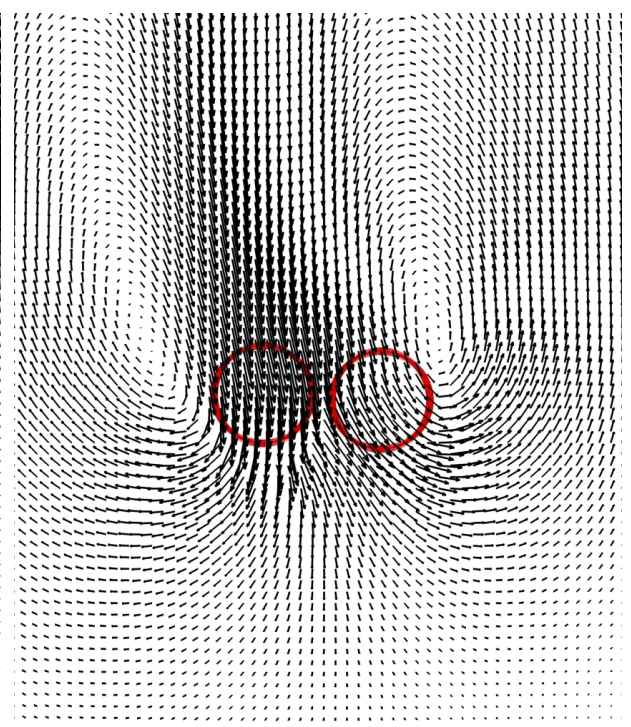

(d) $t=0.8$

Fig. 2: Vector plots of two colliding particles for $S t_{p}=1.18$ - Three different stages of drafting, kissing and tumbling are presented for a small Stokes number case. 


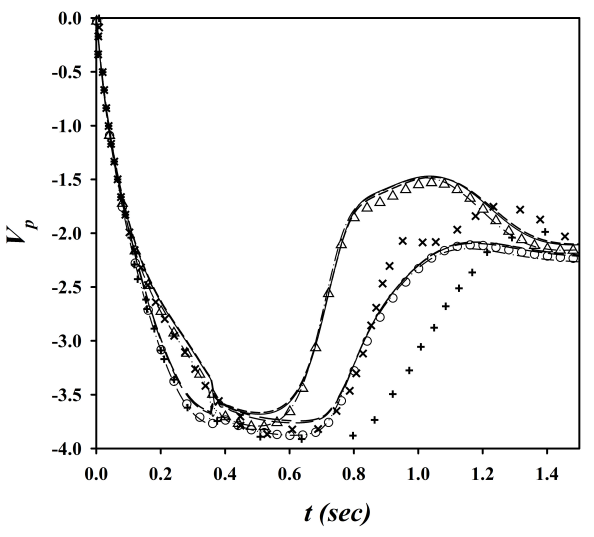

(a) Vertical velocity

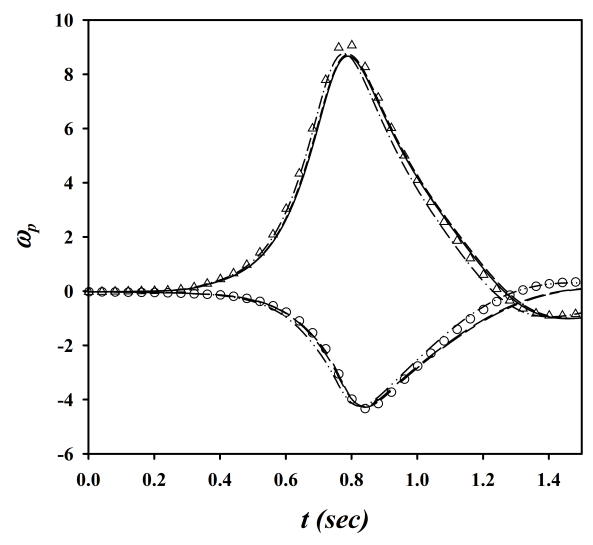

(c) Angular velocity

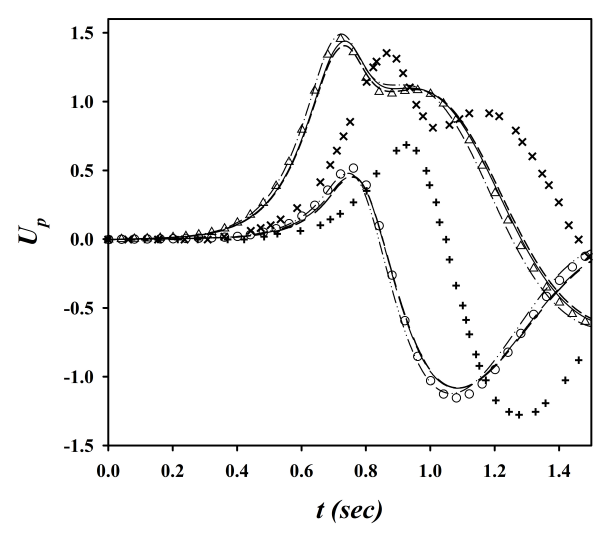

(b) Lateral velocity

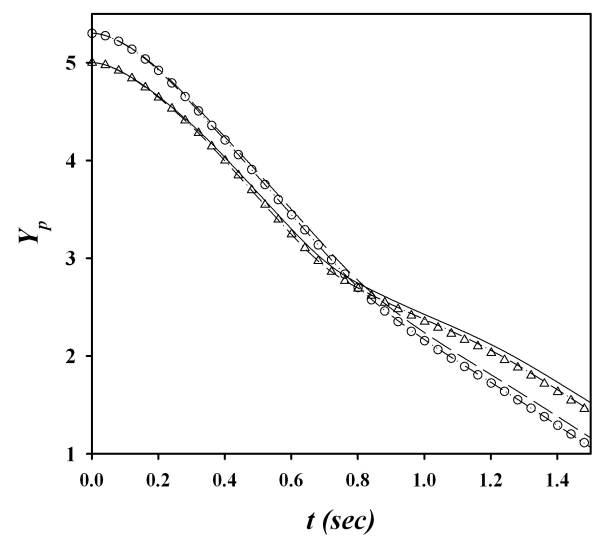

(d) Vertical position

Fig. 3: Linear and angular velocity and vertical position of the two particles at $S t_{p}=1.18$

- Time and grid independence of the collision strategy is tested. — Leading particle $(\Delta t=0.002, h=1 / 256)$, — Trailing particle $(\Delta t=0.002, h=1 / 256)$, --- Leading particle $(\Delta t=0.002, h=1 / 320),-$ - Trailing particle $(\Delta t=0.002, h=1 / 320),-\cdot-\cdot-$ Leading particle $(\Delta t=0.002, h=1 / 192), \cdots \cdots \cdot-$ - Trailing particle $(\Delta t=0.002, h=1 / 192)$, $\triangle$ Leading particle $(\Delta t=0.001, h=1 / 256)$, ○ Trailing particle $(\Delta t=0.001, h=1 / 256)$, $\times$ Leading particle [2], + Trailing particle [2] 


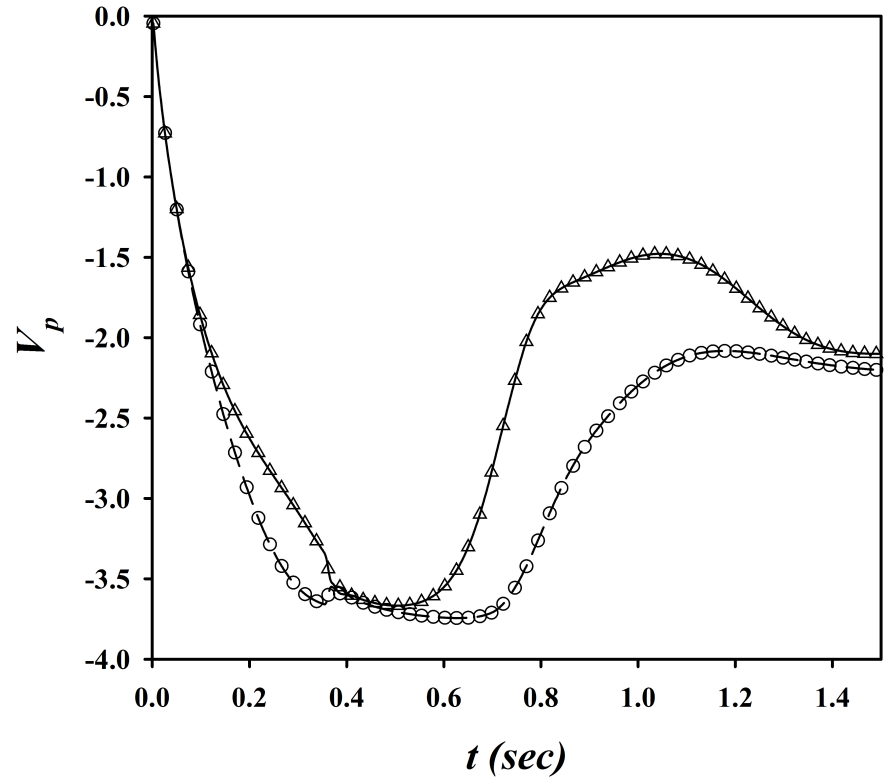

Fig. 4: Independence of the results $(h=1 / 320)$ from the number of sub-steps. - leading particle $N_{k}=5,-$ trailing particle $N_{k}=5, \triangle$ Leading particle $N_{k}=10$, ○ Trailing particle $N_{k}=10$. 

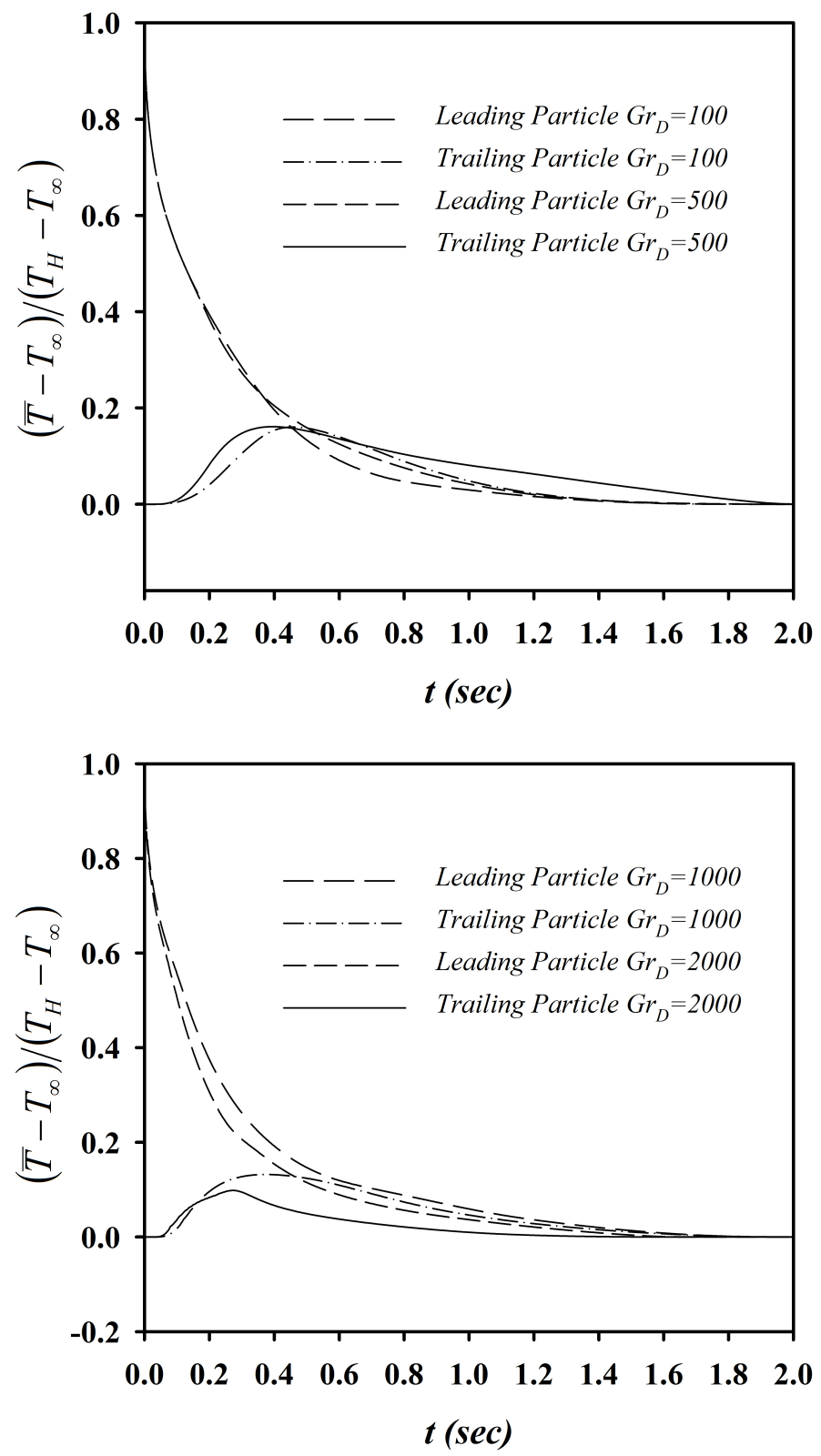

Fig. 5: Time variation of the mean particle temperature for four different Grashof numbers. 


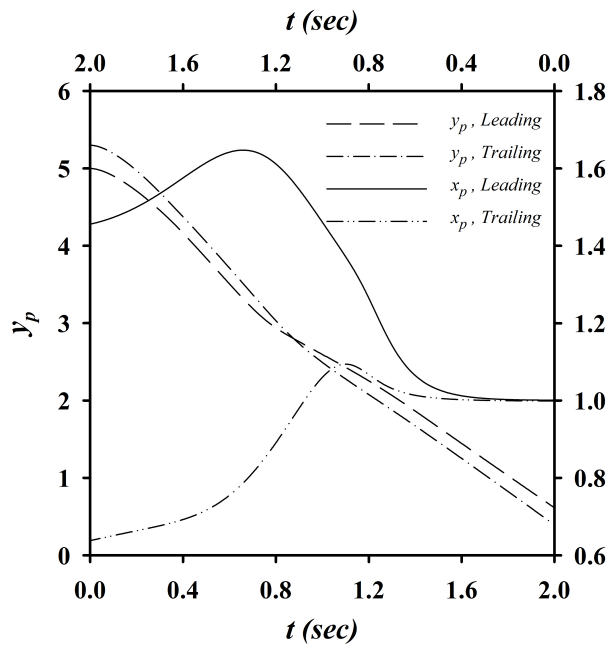

(a) $G r_{D}=100$

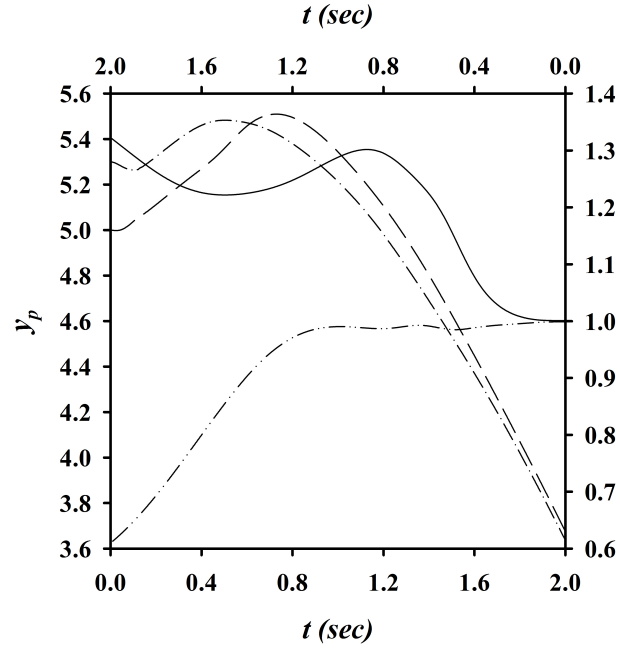

(c) $G r_{D}=1000$

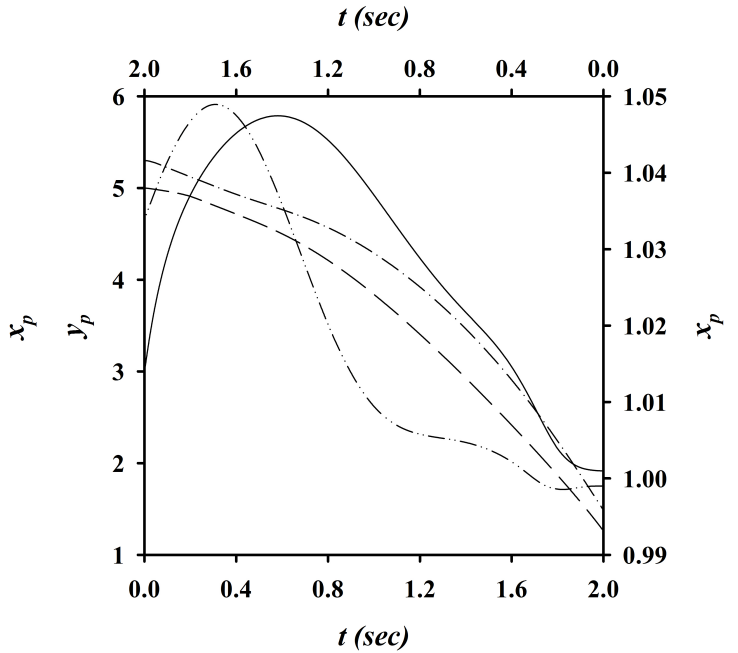

(b) $G r_{D}=500$

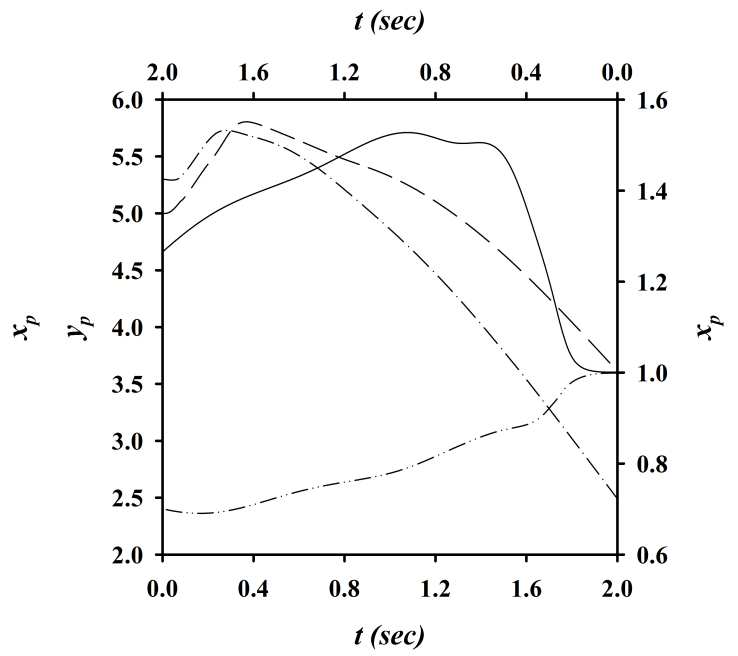

(d) $G r_{D}=2000$

Fig. 6: Time history of the particle positions for four considered Grashof $G r_{D}$ numbers. No tumbling is observed for $G r=500$ and upward collision is observed for $G r=1000$ and $G r=2000$. Horizontal positions $x_{p}$ should be read from the right and top axis and vertical positions $y_{p}$ from the left and bottom axis. 


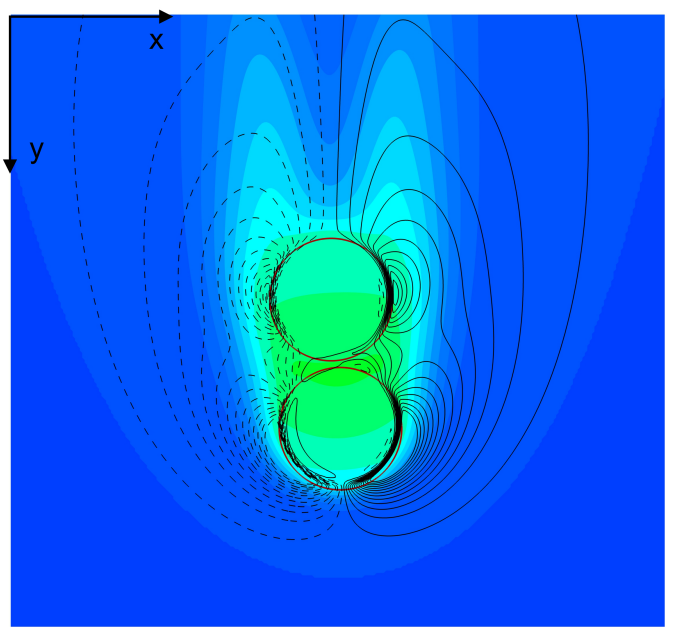

(a) $G r_{D}=100, t=0.45$

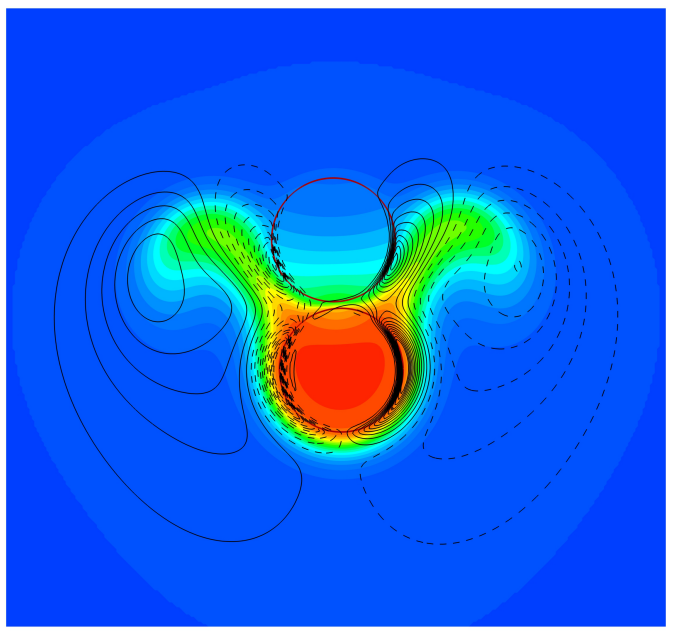

(c) $G r_{D}=1000, t=0.2$

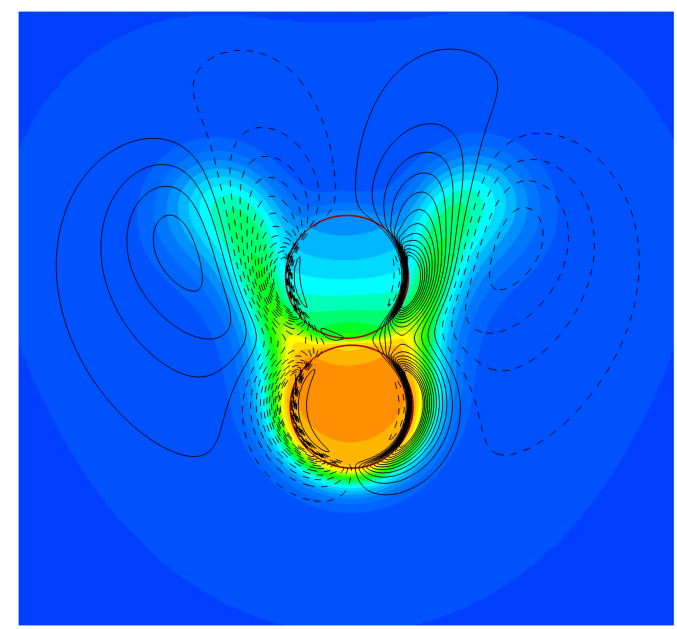

(b) $G r_{D}=500, t=0.25$

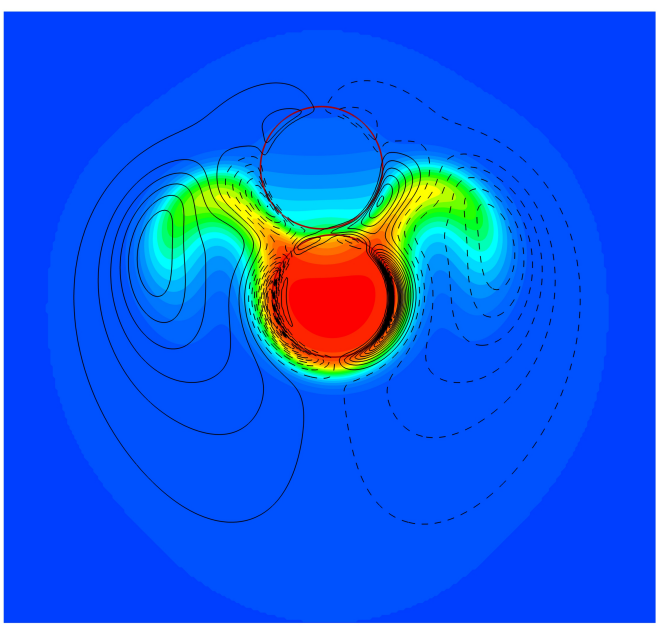

(d) $G r_{D}=2000, t=0.2$

Fig. 7: 26 levels of $\omega_{z}$ are superimposed on 40 levels of temperature contours. Temperature contours are presented for $0<\left(\bar{T}-T_{\infty}\right) /\left(T_{H}-T_{\infty}\right)<0.4$ for all the cases and dashed lines show the negative values. A pair of counter rotating vortices are observed for $G r \geq 500$. 


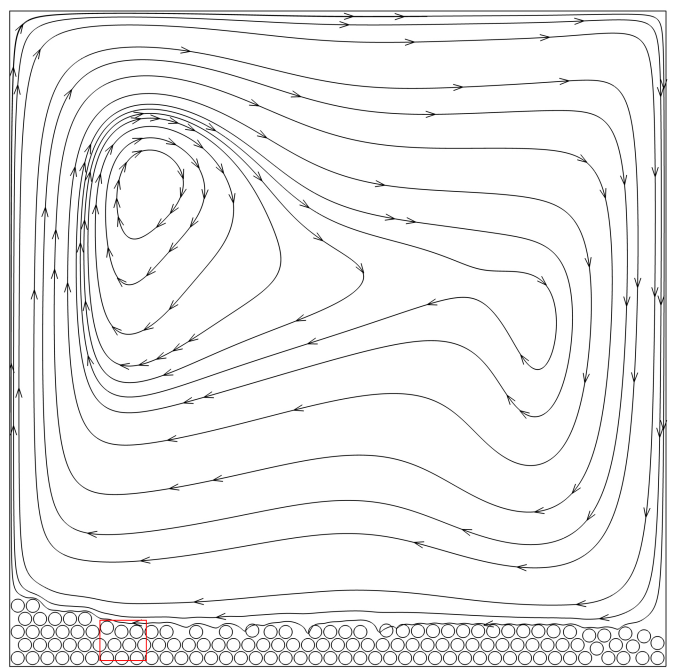

(a) Stream Lines

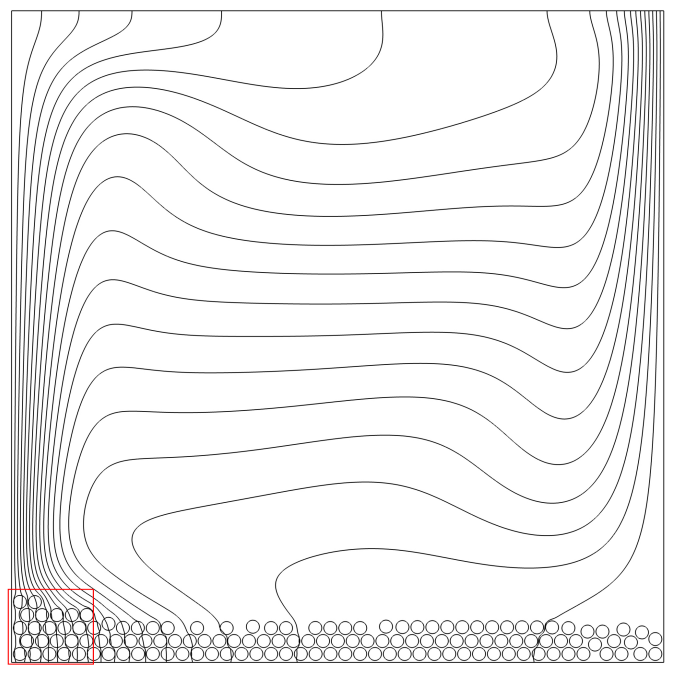

(c) Temperature Contours

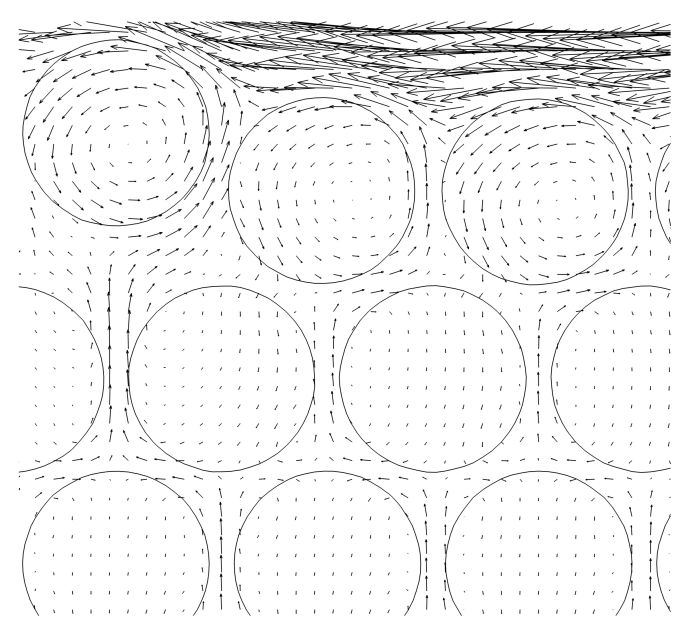

(b) Vector plot

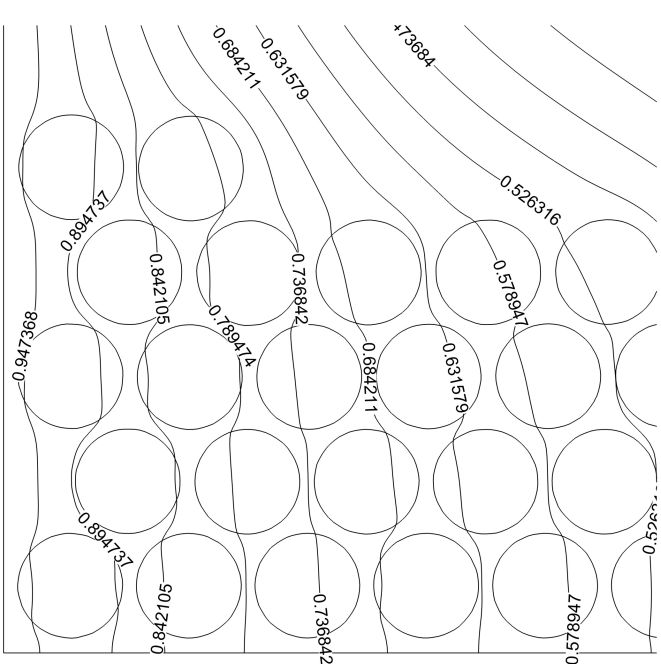

(d) Temperature Contours

Fig. 8: Particle behaviour in the DHC at $G r_{L}=5 \times 10^{5}$ at $t=5$. Magnified vector and contour plots show the particle in a region specified by a box in the corresponding streamline and contour plots. 


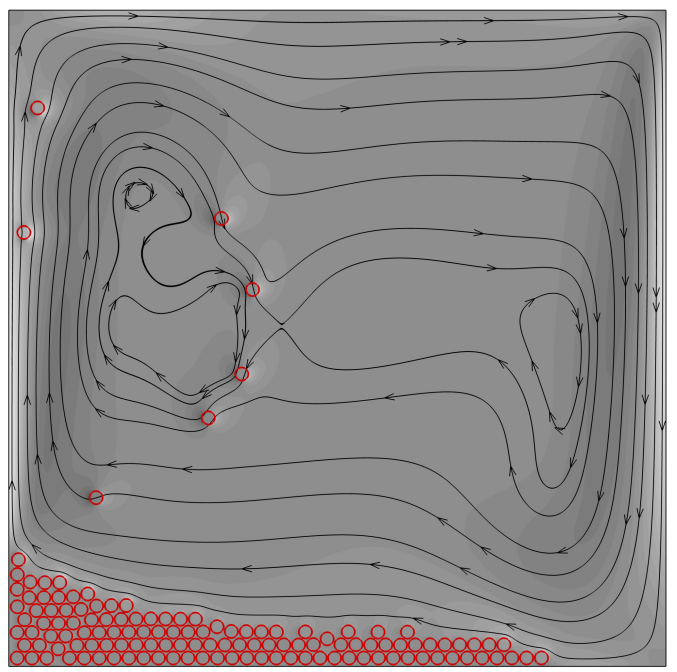

(a) $G r_{L}=10^{6}$

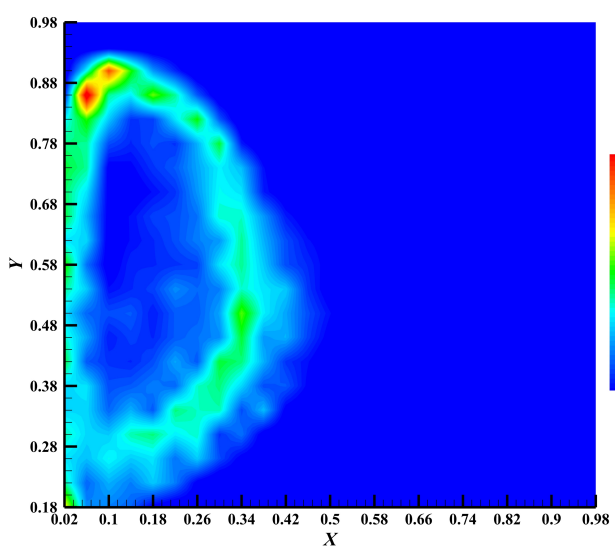

(c) $\bar{N}_{\text {bin }}$ at $G r_{L}=10^{6}$

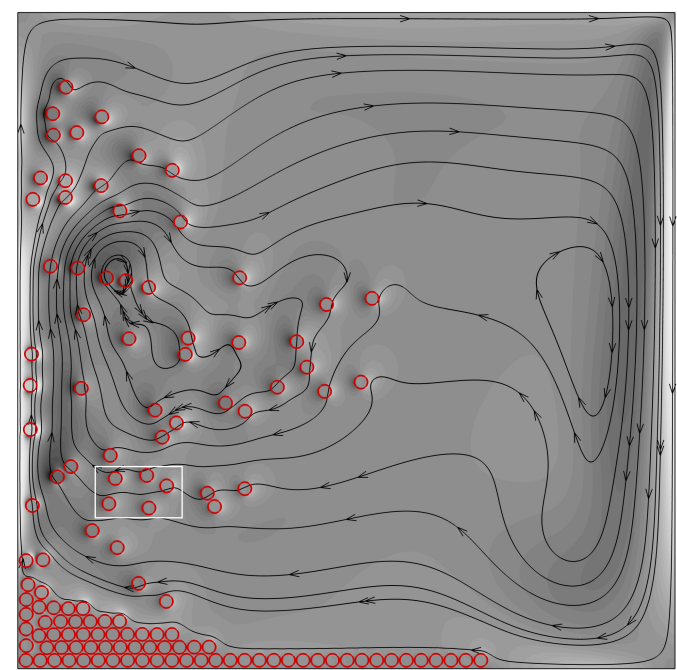

(b) $G r_{L}=2 \times 10^{6}$

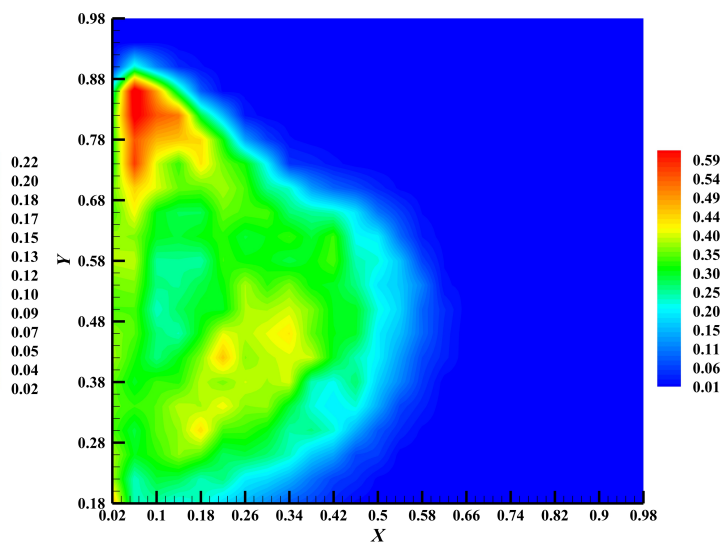

(d) $\bar{N}_{\text {bin }}$ at $G r_{L}=2 \times 10^{6}$

Fig. 9: Particle behaviour in the DHC above the critical Grashof number $G r_{L}=10^{6}$. Vorticity contours and stream lines are plotted at $t_{s}=20$ and the positions are averaged between $t_{0}=8$ and $t_{s}=20$. Position averaging starts at the heigh of the settled particles $y=0.18$ for a better presentation. Vorticity contours are presented between $\omega_{z}=-190$ and $\omega_{z}=240$ in increments of 8.6 . 


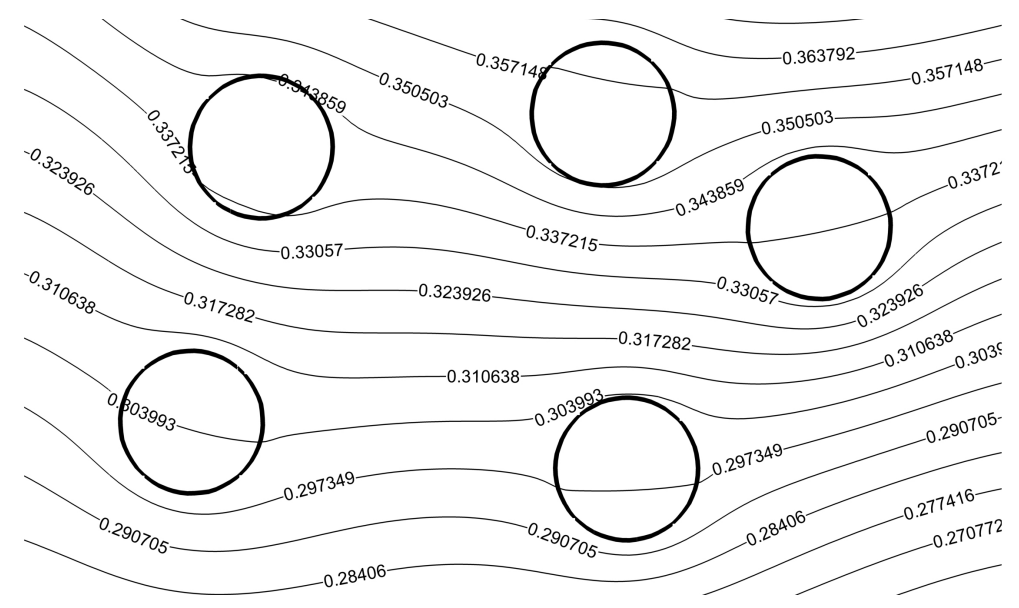

Fig. 10: Temperature contours near particles in the region specified in Figure 9(b) by a box. 

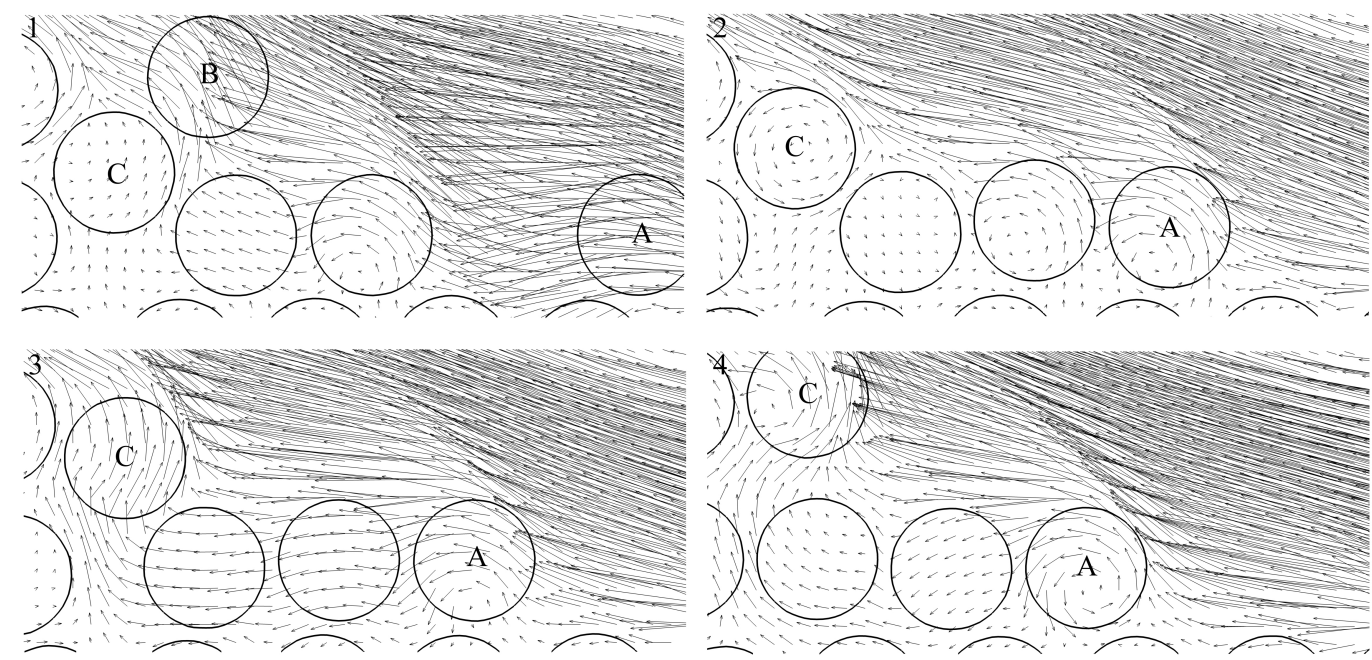

Fig. 11: Escape of a new particle from the bed due to the collision and momentum transfer along the raw of the particles. Frames are labelled $1 \cdots 4$ and $\Delta t$ between the frames is 0.08 starting at $t=20.67$. 


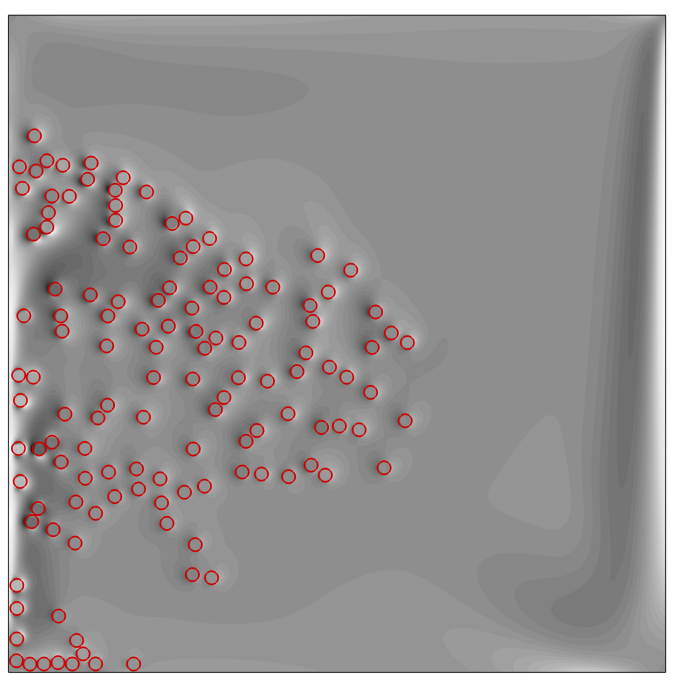

(a) $G r_{L}=5 \times 10^{6}$

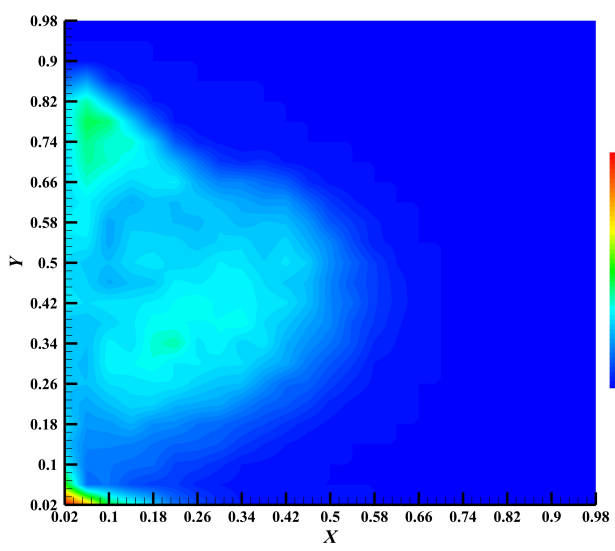

(c) $G r_{L}=5 \times 10^{6}, \bar{N}_{\text {bin }}$

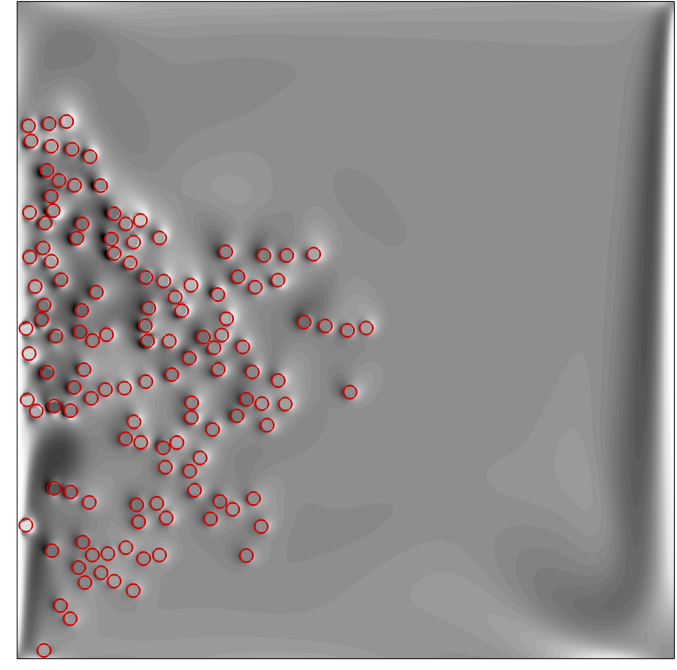

(b) $G r_{L}=10^{7}$

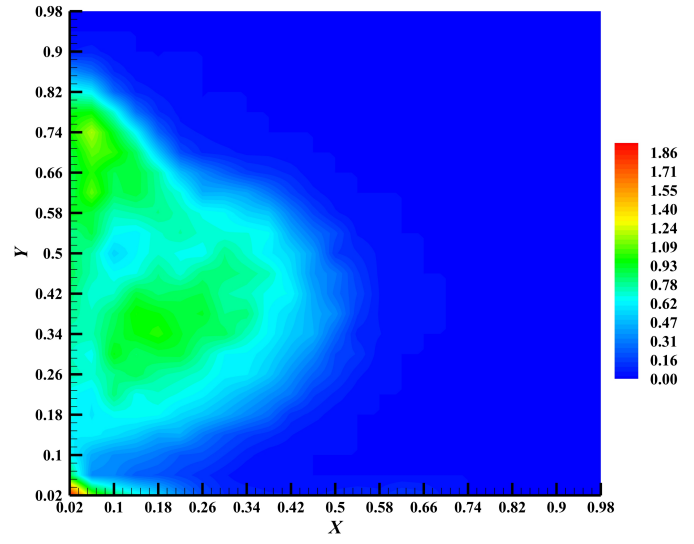

(d) $G r_{L}=10^{7}, \bar{N}_{\text {bin }}$

Fig. 12: Particle behaviour in the DHC above the critical Grashof number $G r_{L}=5 \times 10^{6}$. The positions are averaged between $t_{0}=8$ and $t_{s}=20$ for $G r_{L}=5 \times 10^{6}$ and between $t_{0}=6$ and $t_{s}=18$ for $G r_{L}=10^{7}$ and vorticity contours are presented at $t_{s}$. Vorticity contours are presented between $\omega_{z}=-400$ and $\omega_{z}=500$ in increments of 18 . 


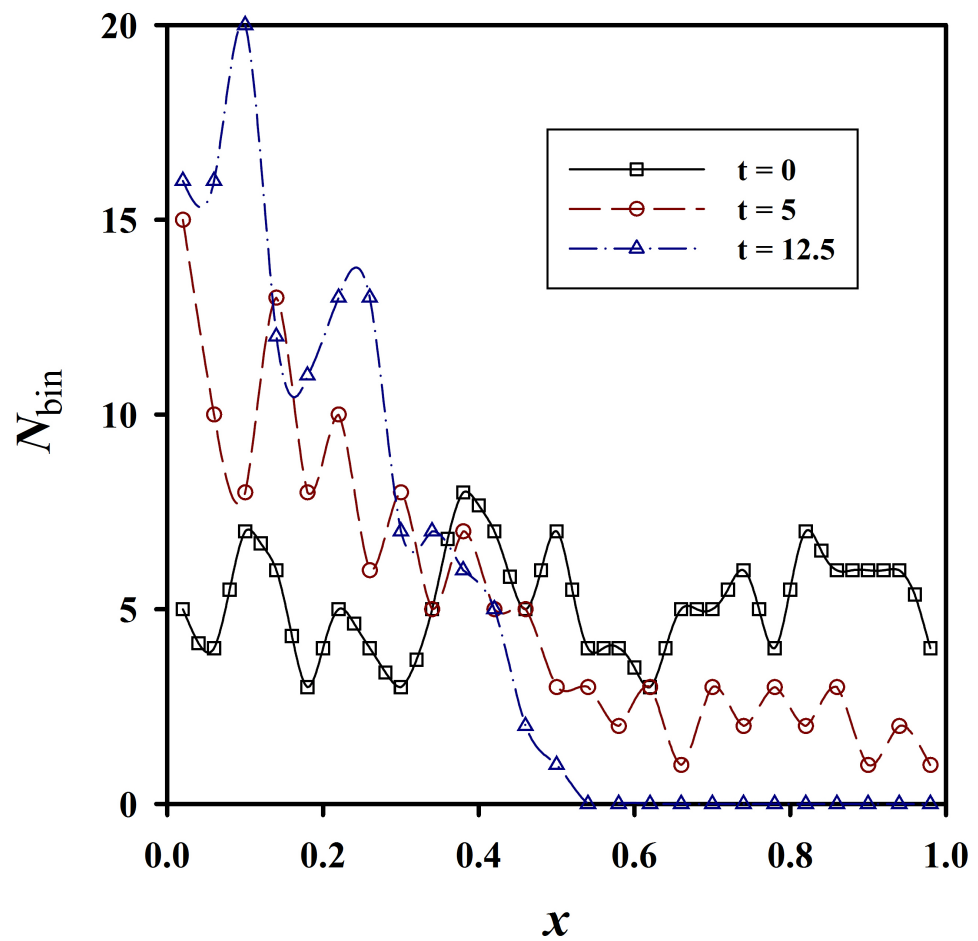

Fig. 13: Instantaneous $N_{\text {bin }}$, for randomly distributed particles in the cavity at $G r_{L}=$ $5 \times 10^{6}$ at three different times. 


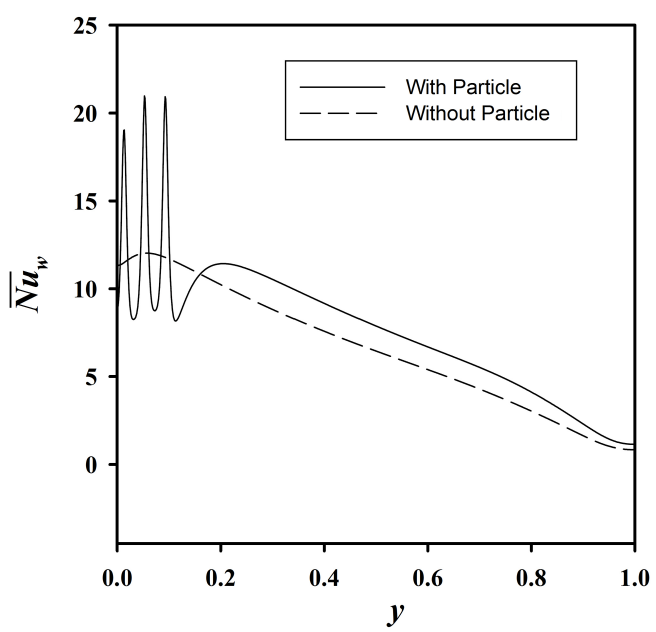

(a) $G r_{L}=5 \times 10^{5}$

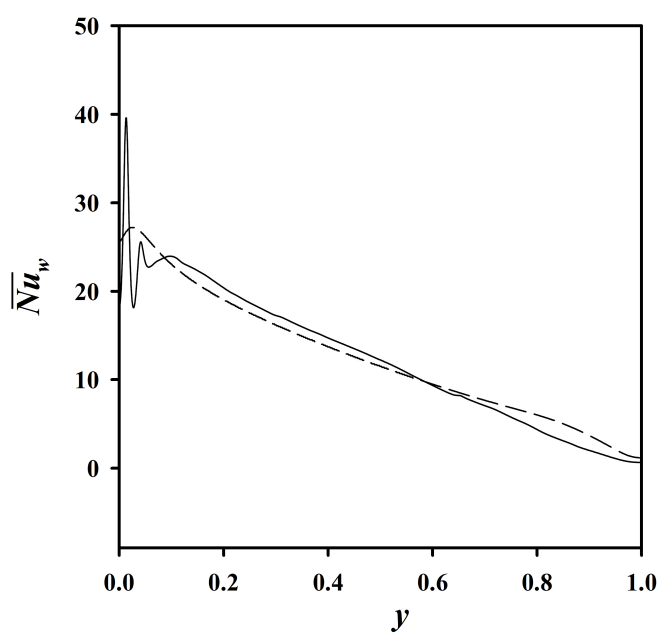

(c) $G r_{L}=5 \times 10^{6}$

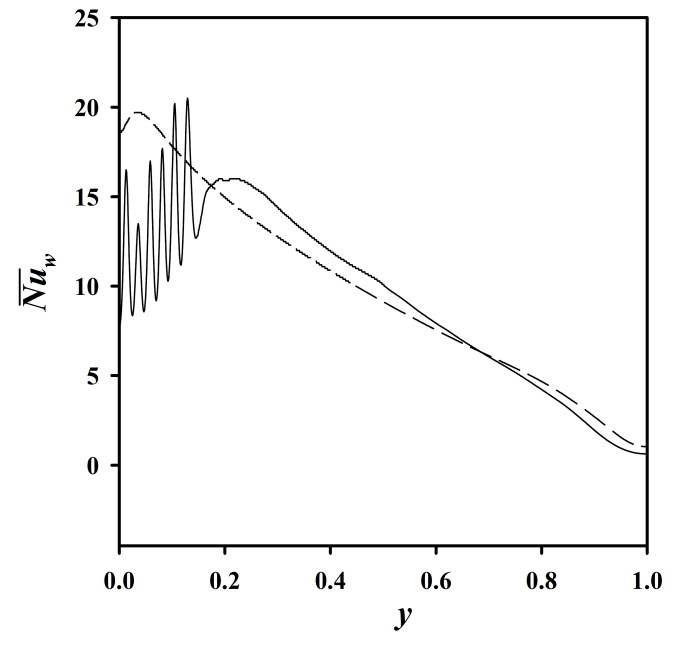

(b) $G r_{L}=2 \times 10^{6}$

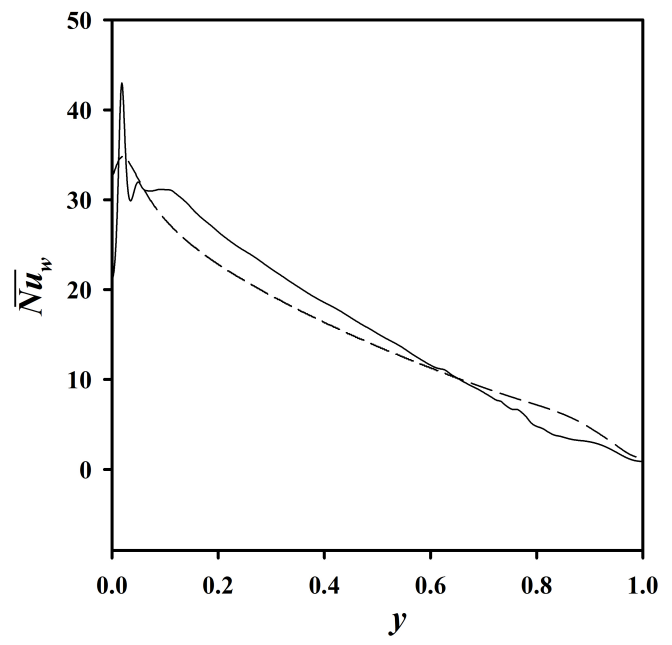

(d) $G r_{L}=10^{7}$

Fig. 14: Variation of the local wall Nusselt number along the hot wall at four different Grashof numbers. 


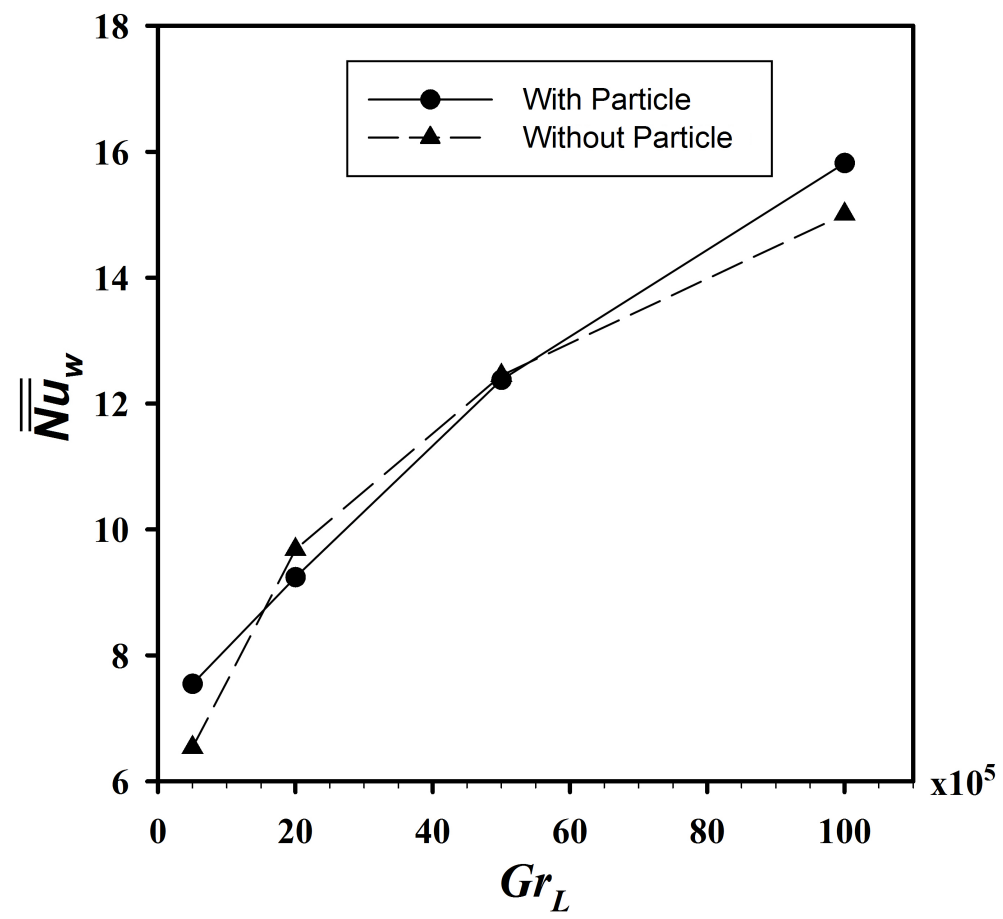

Fig. 15: Particle effects on the wall Nusselt number at different Grashof numbers. 